\title{
Infinite-Energy 2D Statistical Solutions to the Equations of Incompressible Fluids
}

\author{
James P. Kelliher
}

Received: 27 February 2009 / Published online: 14 October 2009

(C) The Author(s) 2009. This article is published with open access at Springerlink.com

\begin{abstract}
We develop the concept of an infinite-energy statistical solution to the Navier-Stokes and Euler equations in the whole plane. We use a velocity formulation with enough generality to encompass initial velocities having bounded vorticity, which includes the important special case of vortex patch initial data. Our approach is to use well-studied properties of statistical solutions in a ball of radius $R$ to construct, in the limit as $R$ goes to infinity, an infinite-energy solution to the Navier-Stokes equations. We then construct an infinite-energy statistical solution to the Euler equations by making a vanishing viscosity argument.
\end{abstract}

Keywords Statistical solutions $\cdot$ Navier-Stokes equations $\cdot$ Euler equations

Mathematics Subject Classification (2000) 76 D06 · 76D05

\section{Introduction}

We develop the concept of a statistical solution to the Navier-Stokes (SSNS) or Euler equations (SSE) in the plane for an important class of velocity fields having sufficient decay of the vorticity at infinity to recover uniquely the velocity field from the vorticity. In particular, this class of velocity fields includes the important case of a vortex patch: a velocity field whose initial vorticity is the characteristic function of a bounded domain.

Our starting point is the velocity formulation of a SSNS on a bounded domain given by Foias in [5,6]. (A highly accessible account of the theory of SSNSs is given in [7], to which we refer often.) We adapt this formulation slightly, of necessity changing the energy equality and using the same class of test functions as for homogeneous solutions in the plane (Sect. 6). Our definition of an infinite-energy SSE is the same with the viscosity set

J. P. Kelliher $(\bowtie)$

Department of Mathematics, University of California, 900 University Ave,

Riverside, CA 92521, USA

e-mail: kelliher@math.ucr.edu 
to zero. We construct our infinite-energy SSNS by showing that it is the limit, in a special sense, of a sequence of statistical solutions on balls of radius $R$ as $R \rightarrow \infty$. At the core of our approach is the expanding domain limit for deterministic solutions to the Navier-Stokes equations established in [10], extended to handle infinite-energy solutions. We then construct our infinite-energy SSE by making a vanishing viscosity argument.

In the deterministic setting, the infinite-energy solutions that we consider correspond to an initial velocity lying in the space $E_{m}$ of $[2,3]$. A vector $v$ belongs to $E_{m}$ if it is divergence-free and can be written in the form $v=\sigma+v^{\prime}$, where $v^{\prime}$ is in $L^{2}\left(\mathbb{R}^{2}\right)$ and where $\sigma$ is a smooth stationary solution to the Euler equations whose vorticity is radially symmetric and compactly supported (see Sect. 2 for more details). A unique global solution to the Navier-Stokes equations exists and remains in the space $E_{m}$ for all time as long as the forcing has finite energy. The same can be said of solutions to the Euler equations if one imposes restrictions on the initial vorticity; for instance, that it lie in $L^{1} \cap L^{\infty}$. This encompasses the case of a classical vortex patch-initial vorticity that equals the characteristic function of a bounded domain (see also Corollary 2.3).

A key parameter of any vortex patch is the total mass of its vorticity,

$$
m=\int_{\mathbb{R}^{2}} \omega .
$$

Only when $m=0$ will the velocity field have finite energy (lie in $L^{2}$ ), which excludes the case of a classical vortex patch. In a sense, $m$ measures how infinite the energy is.

When working with statistical solutions one would like to allow $m$ to take on different values, because classical vortex patches that are nearly identical will typically have different values of $m$. Thus, we need to consider the spaces $E_{m}$ for all values of $m$ simultaneously.

We give a velocity rather than a vorticity formulation of our statistical solutions for several reasons. First, a vorticity formulation would require imposing higher regularity on the initial vorticity than required for solutions to the Navier-Stokes equations: it would be technically quite difficult to assume anything weaker than the initial vorticity lying in $L^{1}$, as in [1]. Second, it would be hard to obtain convergence of the vorticity in the vanishing viscosity limit, even with higher regularity of the initial data, without knowing that the velocity decays at infinity, and this does not come from the Biot-Savart law. Third, a start in this direction has already been made in [4] for time-independent solutions to damped and driven Navier-Stokes and Euler equations in the vorticity formulation.

Constantin and Ramos do not specifically address infinite-energy solutions in [4]; however, their definition of such a solution requires no change at all to encompass infinite-energy solutions and neither does their proof of the vanishing viscosity limit. Their construction of a stationary statistical solution to the Navier-Stokes equations as a long-time average of a deterministic solution to the damped and driven Navier-Stokes equations does assume finite energy. Nothing deep need be done, however, to extend their construction to allow infinite energy solutions (and so allow initial vortex patch data): one need only assume infinite-energy forcing.

This paper is organized as follows: In Sect. 2, we define the function spaces in which we will work. We characterize the projection operator we will use to construct the initial velocities in $\Omega_{R}$ in Sect. 3. In Sect. 4, we define weak deterministic solutions to the NavierStokes and Euler equations and give the basic well-posedness and regularity results for such solutions. The deterministic expanding domain limit of [10] is established for infinite-energy solutions in Sect. 5. We give the definition of a statistical solution to the Navier-Stokes equations in velocity form, for finite as well as infinite energy, in Sect. 6, and construct an infinite-energy statistical solution to the Navier-Stokes equations in Sect. 7, showing that it 
is unique. In Sect. 8 we construct an infinite-energy statistical solution to the Euler equations using a vanishing viscosity argument.

\section{Function Spaces and the Biot-Savart Law}

Let

$$
\Omega_{R}=\text { the disk of radius } R \text { centered at the origin, }
$$

with $\Omega_{\infty}=\mathbb{R}^{2}$, and define the classical function spaces of incompressible fluid mechanics,

$$
\begin{aligned}
H_{R} & =H\left(\Omega_{R}\right)=\left\{u \in\left(L^{2}\left(\Omega_{R}\right)\right)^{2}: \operatorname{div} u=0, u \cdot \mathbf{n}=0 \text { on } \partial \Omega_{R}\right\}, \\
V_{R} & =V\left(\Omega_{R}\right)=\left\{u \in\left(H^{1}\left(\Omega_{R}\right)\right)^{2}: \operatorname{div} u=0, u=0 \text { on } \partial \Omega_{R}\right\}, \\
H & =H_{\infty}=H\left(\mathbb{R}^{2}\right), \quad V=V_{\infty}=V\left(\mathbb{R}^{2}\right) .
\end{aligned}
$$

We endow $H_{R}$ with the $L^{2}$-norm. For $V_{R}$, we use the $H^{1}$-norm:

$$
\|u\|_{V_{R}}=\|u\|_{L^{2}\left(\Omega_{R}\right)}+\|\nabla u\|_{L^{2}\left(\Omega_{R}\right)} .
$$

Note, in particular, that

$$
\|u\|_{L^{2}\left(\Omega_{R}\right)} \leq\|u\|_{V_{R}}, \quad\|\nabla u\|_{L^{2}\left(\Omega_{R}\right)} \leq\|u\|_{V_{R}} .
$$

Had we used the Poincare inequality to replace Eq. 2.1 with the equivalent norm that includes only the second term, as is normally done for a bounded domain, it would have introduced a factor of $R$ in the right-hand side of the first inequality, preventing us from having a consistent norm with which to compare solutions on $\Omega_{R}$ for different values of $R$.

Our deterministic infinite-energy solutions will lie in the space $E_{m}$ of [3]. A vector $v$ belongs to $E_{m}$ if it is divergence-free and can be written in the form $v=\sigma+v^{\prime}$, where $v^{\prime}$ is in $L^{2}\left(\mathbb{R}^{2}\right)$ and where $\sigma$ is a stationary vector field, meaning that $\sigma$ is of the form,

$$
\sigma=\left(-\frac{x^{2}}{r^{2}} \int_{0}^{r} \rho g(\rho) d \rho, \frac{x^{1}}{r^{2}} \int_{0}^{r} \rho g(\rho) d \rho\right)
$$

with $g$ in $C_{0}^{\infty}(\mathbb{R})$. The subscript $m \in \mathbb{R}$ is the integral over all space of the vorticity,

$$
\omega(v)=\partial_{1} v^{2}-\partial_{2} v^{1}
$$

$E_{m}$ is an affine space; fixing an origin, $\sigma$, in $E_{m}$ we can define a "norm" by $\left\|\sigma+v^{\prime}\right\|_{E_{m}}=$ $\left\|v^{\prime}\right\|_{L^{2}(\Omega)}$. Convergence in $E_{m}$ is equivalent to convergence in the $L^{2}$-norm to a vector in $E_{m}$.

We will find it convenient to fix a choice of origin for $E_{m}$ as follows. For $E_{1}$ we choose $\sigma_{1}$ of the form Eq. 2.3 with $\omega\left(\sigma_{1}\right)$ supported in the unit disk and with

$$
\int_{\Omega_{1}} \omega\left(\sigma_{1}\right)=\int_{\mathbb{R}^{2}} \omega\left(\sigma_{1}\right)=1
$$

We then use $\sigma_{m}=m \sigma_{1}$ as the origin for $E_{m}$.

Let $\psi_{\sigma_{1}}$ be a given fixed stream function for $\sigma_{1}$. As in [11], $\psi_{\sigma_{1}}$ is radially symmetric with

$$
\psi_{\sigma_{1}}(x)=C_{2}+\frac{1}{2 \pi} \log |x|
$$


for all $|x| \geq 1$. For $|x| \geq 1,\left|\sigma_{1}(x)\right|=1 /|x|$ by Eq. 2.3, and

$$
\begin{aligned}
\left\|\sigma_{1}\right\|_{H^{1}\left(\Omega_{R} \backslash \Omega_{R-1}\right)}^{2} & =2 \pi \int_{R-1}^{R} \frac{1}{r^{2}} r d r+2 \pi \int_{R-1}^{R} \frac{1}{r^{4}} r d r \\
& =2 \pi \log (R /(R-1))+\pi\left[(R-1)^{-2}-R^{-2}\right] \rightarrow 0 \text { as } R \rightarrow \infty .
\end{aligned}
$$

Equation 2.4 also gives $\Delta \sigma_{1}=\Delta \nabla^{\perp} \psi_{\sigma_{1}}=\nabla^{\perp} \Delta \psi_{\sigma_{1}}=0$ on $\Omega_{1}^{C}$.

The spaces $H_{R}$ and $E_{m}$, or $V_{R}$ and $E_{m} \cap \dot{H}^{1}\left(\mathbb{R}^{2}\right)$, where $\dot{H}^{1}\left(\mathbb{R}^{2}\right)$ is the set of all functions whose gradient lies in $L^{2}\left(\mathbb{R}^{2}\right)$, are the appropriate ones for initial velocities for weak deterministic solutions to the Navier-Stokes equations, but for the Euler equations more regularity is required to obtain well-posedness. Rather than being as general as possible, we will assume that for deterministic solutions the initial vorticity lies in $L^{\infty}$ for solutions on $\Omega_{R}$ and in $L^{p_{0}} \cap L^{\infty}$, for some $p_{0}<2$ for solutions on $\mathbb{R}^{2}$. Slightly unbounded vorticities could be handled, as in [10], with little complication. This gives not only existence but uniqueness of the solutions. (The uniqueness of solutions for bounded initial vorticity is due to Yudovich [14], as is the uniqueness for unbounded vorticities [15].)

Thus, we fix $p_{0}<2$, and define the spaces

$$
\mathbb{Y}_{m}=\left\{u \in E_{m}: \omega(u) \in L^{p_{0}} \cap L^{\infty}\right\}
$$

with "norm"

$$
\|u\|_{\mathbb{Y}_{m}}=\|u\|_{E_{m}}+\left\|\omega\left(u-\sigma_{m}\right)\right\|_{L^{p_{0} \cap L^{\infty}}}
$$

and

$$
\mathbb{Y}\left(\Omega_{R}\right)=\left\{u \in H\left(\Omega_{R}\right) \cap H^{1}\left(\Omega_{R}\right): \omega(u) \in L^{\infty}\right\}
$$

with norm

$$
\|u\|_{\mathbb{Y}\left(\Omega_{R}\right)}=\|u\|_{H^{1}\left(\Omega_{R}\right)}+\|\omega(u)\|_{L^{p_{0} \cap L^{\infty}\left(\Omega_{R}\right)}}, \quad R<\infty .
$$

Because $L^{p_{0}}\left(\Omega_{R}\right) \subseteq L^{\infty}\left(\Omega_{R}\right)$, using only the $L^{\infty}$-norm of $\omega(u)$ in the $Y\left(\Omega_{R}\right)$-norm would give a simpler, equivalent norm. We avoid doing this, however, for the same reason we avoided the use of Poincare's inequality in defining the $V_{R}$-norm in Eq. 2.1.

For statistical solutions in the whole plane, we do not want to assume that the value of $m$ is fixed, so we must deal with larger spaces. For statistical solutions to the Navier-Stokes equations we will use

$$
\mathbb{E}=\bigcup_{m \in \mathbb{R}} E_{m} \text { and } \mathbb{E}^{1}=\bigcup_{m \in \mathbb{R}} E_{m} \cap \dot{H}^{1}\left(\mathbb{R}^{2}\right)
$$

and for statistical solutions to the Euler equations we will use

$$
\mathbb{Y}=\bigcup_{m \in \mathbb{R}} \mathbb{Y}_{m}
$$

$\mathbb{E}, \mathbb{Y}$, and $\mathbb{E}^{1}$ are function spaces, being closed under addition, with the norms

$$
\begin{aligned}
\left\|\sigma_{m}+v\right\|_{\mathbb{E}} & =|m|+\|v\|_{L_{2}}, \quad\|u\|_{\mathbb{Y}}=\|u\|_{\mathbb{E}}+\|\omega(u)\|_{L^{p_{0} \cap L^{\infty}}}, \\
\left\|\sigma_{m}+v\right\|_{\mathbb{E}^{1}} & =\left\|\sigma_{m}+v\right\|_{\mathbb{E}}+\|\nabla v\|_{L^{2}\left(\mathbb{R}^{2}\right)} .
\end{aligned}
$$

These norms induce metrics on their respective spaces. Because $H$ and $V$ are separable, so too are $\mathbb{E}$ and $\mathbb{E}^{1}$. The space $\mathbb{Y}$, however, is not separable, because $L^{\infty}\left(\mathbb{R}^{2}\right)$ is not. 
There exists a unique decomposition of any $u$ in $\mathbb{E}, \mathbb{Y}$, or $\mathbb{E}^{1}$ of the form $u=\sigma_{m}+v, m \in$ $\mathbb{R}, v \in H$. Given such a $u$, we define

$$
m(u)=m, \quad \sigma(u)=\sigma_{m(u)} .
$$

Definition 2.1 We say that the support of a measure $\mu$ on the function space $X$ is bounded in $X$ if

$$
\operatorname{supp} \mu \subseteq\left\{u \in X:\|u\|_{X} \leq M\right\} \text { for some } M<\infty .
$$

If $Y$ is a subspace of $X$, we say that the support of a measure $\mu$ is $(X, Y)$-bounded if

$$
\operatorname{supp} \mu \subseteq\left\{u \in Y:\|u\|_{X} \leq M\right\} \text { for some } M<\infty .
$$

That is, the support of $\mu$ lies in the subspace, but only its norm in the full space is controlled.

Lemma 2.2 [Biot-Savart law] Let $p$ be in $[1,2)$ and let $q>2 p /(2-p)$. For any vorticity $\omega$ in $L^{p}\left(\mathbb{R}^{2}\right)$ there exists a unique divergence-free vector field $u$ in $L^{p}\left(\mathbb{R}^{2}\right)+L^{q}\left(\mathbb{R}^{2}\right)$ whose curl is $\omega$, with $u$ being given by the Biot-Savart law,

$$
u=K * \omega .
$$

Here, $K$ is the Biot-Savart kernel, $K(x)=(1 / 2 \pi) x^{\perp} /|x|^{2}$.

Proof See the proof of Proposition 3.1.1 pp. 44-45 of [3].

Corollary 2.3 For any vorticity $\omega$ in $L^{1} \cap L^{\infty}\left(\mathbb{R}^{2}\right)$ there exists a unique divergence-free vector field $u$ in $L^{\infty}\left(\mathbb{R}^{2}\right)$ whose curl is $\omega$, with $u$ given by Eq. 2.8. If $\omega$ is also compactly supported then $u$ lies in $E_{m}$, where $m=\int_{\mathbb{R}^{2}} \omega$.

Proof By Lemma 2.2 applied with $p=1, K * \omega$ is the unique vector field in $L^{1}+L^{\infty}$ whose vorticity is $\omega$. But also,

$$
\begin{aligned}
\|K * \omega\|_{L^{\infty}} & \leq\left\|\left(\chi_{\Omega_{1}} K\right) * \omega\right\|_{L^{\infty}}+\left\|\left(1-\chi_{\Omega_{1}}\right) K * \omega\right\|_{L^{\infty}} \\
& \leq\left\|\chi_{\Omega_{1}} K\right\|_{L^{1}}\|\omega\|_{L^{\infty}}+\left\|\left(1-\chi_{\Omega_{1}}\right) K\right\|_{L^{\infty}}\|\omega\|_{L^{1}} \\
& \leq C\|\omega\|_{L^{1} \cap L^{\infty}} .
\end{aligned}
$$

Here, $\chi_{A}$ is the characteristic function of $A$. This shows that, in fact, $K * \omega$ is in $L^{\infty}$ and is the unique such vector field. The last statement in the corollary follows from Lemma 1.3.1 of [3].

\section{Projection Operators}

Let $\mathbf{P}_{V_{R}}: \mathbb{E}^{1} \rightarrow V_{R}$ be restriction to $\Omega_{R}$ followed by projection onto $V_{R} . \mathbf{P}_{V_{R}}$ is well-defined because as Hilbert spaces $V_{R}$ is a closed subspace of $H_{d i v}^{1}\left(\Omega_{R}\right)$, the space of all divergencefree vector fields in $\left(H^{1}\left(\Omega_{R}\right)\right)^{2}$, endowed with the inner product,

$$
\langle u, v\rangle_{H_{d i v}^{1}\left(\Omega_{R}\right)}=(u, v)+(\nabla u, \nabla v) .
$$

We can describe $\mathbf{P}_{V_{R}}$ explicitly by characterizing $V_{R}^{\perp}$, the orthogonal complement of $V_{R}$ in $H_{d i v}^{1}\left(\Omega_{R}\right)$. By definition, $w$ is in $V_{R}^{\perp}$ if and only if $\langle w, v\rangle_{H_{d i v}^{1}\left(\Omega_{R}\right)}=0$ for all $v$ in $V_{R}$. 
Treating $\Delta w$ as a distribution, integrating by parts gives $(w-\Delta w, v)=0$ for all $v$ in $\mathcal{V}_{R}$, where $\mathcal{V}_{R}=V_{R} \cap \mathcal{D}\left(\Omega_{R}\right)$. It follows from this that $w$ is in $V_{R}^{\perp}$ if and only if

$$
\Delta w-w=\nabla p
$$

for some $p$ in $L^{2}(\Omega)$ (see, for instance, Proposition I.1.1 of [13]) and, of course, div $w=0$.

Now let $u$ lie in $H_{d i v}^{1}\left(\Omega_{R}\right)$ and let $\bar{u}=\mathbf{P}_{V_{R}} u$. Then $w=u-\bar{u}$ lies in $V_{R}^{\perp}$ so from Eq. 3.1,

$$
\begin{cases}\Delta \bar{u}-\bar{u}=\Delta u-u+\nabla p & \text { in } \Omega_{R}, \\ \operatorname{div} \bar{u}=\Delta p=0 & \text { in } \Omega_{R}, \\ \bar{u}=0 & \text { on } \partial \Omega_{R} .\end{cases}
$$

Equality is to hold in a weak sense in Eq. 3.2. Since $u$ is in $H^{1}$, however, $f=\Delta u-u+\bar{u}$ is in $H^{-1}$, which is sufficient to conclude that $\bar{u}$ is in $V$ and $p$ is in $L^{2}$ (see, for instance, Remark I.2.6 of [13]). Also, the solution to Eq. 3.2 is unique because otherwise it would follow that -1 is an eigenvalue of the Stokes operator, $-\mathcal{P} \Delta$, where $\mathcal{P}$ is the Leray projector. But the Stokes operator is positive-definite, so all its eigenvalues are positive.

The estimates involving the operator $\mathbf{P}_{V_{R}}$ are hard to prove directly using this characterization. It is simpler to employ an approximate projection operator $\mathbf{U}_{R}$, and use the fact that projection into $V_{R}$ gives the closest vector field in $V_{R}$ to the vector field being projected. (The same idea is used for projection into $H_{R}$ in $[8,11]$.)

To define $\mathbf{U}_{R}$ we need two cutoff functions, $\varphi_{R}$ and $h_{R}$.

Let $\varphi_{1}$ in $C^{\infty}\left(\Omega_{1}\right)$ take values in $[0,1]$ and be defined so that $\varphi_{1}=1$ on $\Omega_{1 / 2}$ and so that $\varphi_{1}$ and $\nabla \varphi_{1}$ are both zero on $\partial \Omega_{R}$. Let $\varphi_{R}(\cdot)=\varphi_{1}(\cdot / R)$. Observe that $\varphi_{R}$ and $\nabla \varphi_{R}$ both vanish on $\partial \Omega_{R}$.

Let $g$ in $C^{\infty}([0,3 / 4])$ taking values in $[0,1]$ be defined so that $g(0)=g^{\prime}(0)=0$ and $g=1$ on $[1 / 2,1]$. Then define $h_{R}$ in $C^{\infty}\left(\Omega_{R}\right)$ by $h_{R}(x)=g(R-|x|)$ for points $x$ in $\Omega_{R} \backslash \Omega_{R-1}$ and $h_{R}=1$ on $\Omega_{R-1}$. Observe that

$$
\left\|h_{R}\right\|_{C^{k}} \leq C_{k},
$$

$k=0,1, \ldots$, for constants $C_{k}$ independent of $R$ in $[1, \infty)$. Also, $h_{R}=0$ and $\nabla h_{R}=0$ on $\partial \Omega_{R}$.

Definition 3.1 Define $\mathbf{U}_{R}: \mathbb{E}^{1} \rightarrow V_{R}$ by

$$
\mathbf{U}_{R}(u)=\nabla^{\perp}\left(h_{R}\left(\psi_{\sigma_{m}}-\psi_{\sigma_{m}}(R)\right)\right)+\nabla^{\perp}\left(\varphi_{R} \psi_{v}\right)
$$

for $u=\sigma_{m}+v$ in $E_{m}$. Here, $\psi_{v}$ is the stream function for $v$ chosen so that $\int_{\Omega_{R}} \psi_{v}=0$ on $\partial \Omega_{R}$.

Lemma 3.2 $\mathbf{P}_{V_{R}}$ maps $\mathbb{E}^{1}$ continuously onto $V_{R}$ with

$$
\left\|u-\mathbf{P}_{V_{R}} u\right\|_{H^{1}\left(\Omega_{R}\right)} \leq C\|u-\sigma(u)\|_{H^{1}\left(\Omega_{R} \backslash \Omega_{R / 2}\right)}+|m(u)| \beta(R),
$$

where

$$
\beta(R)=\left\|\sigma_{1}\right\|_{H^{1}\left(\Omega_{R} \backslash \Omega_{R-1}\right)} \rightarrow 0 \text { as } R \rightarrow \infty,
$$

and

$$
\begin{aligned}
\left\|\mathbf{P}_{V_{R}}\left(u-\sigma_{m}\right)\right\|_{V_{R}} & \leq\left\|u-\sigma_{m}\right\|_{V} \\
\left\|\mathbf{P}_{V_{R}} u\right\|_{V_{R}} & \leq\|u\|_{\mathbb{E}^{1}}+C|m(u)| .
\end{aligned}
$$


Proof That $\mathbf{P}_{V_{R}}$ maps onto $V_{R}$ is clear, and it is continuous because the restriction and the projection operators are continuous.

To prove Eq. 3.4, let $u=\sigma_{m}+v$ in $E_{m} \cap \dot{H}^{1}\left(\mathbb{R}^{2}\right)$. Then

$$
\left\|u-\mathbf{U}_{R} u\right\|_{H^{1}\left(\Omega_{R}\right)} \leq\left\|\sigma_{m}-\mathbf{U}_{R} \sigma_{m}\right\|_{H^{1}\left(\Omega_{R}\right)}+\left\|v-\mathbf{U}_{R} v\right\|_{H^{1}\left(\Omega_{R}\right)} .
$$

It follows from the proof of Lemma 4.2 of [10] that

$$
\left\|v-\mathbf{U}_{R} v\right\|_{H^{1}\left(\Omega_{R}\right)} \leq C\|u-\sigma(u)\|_{H^{1}\left(\Omega_{R} \backslash \Omega_{R / 2}\right)} .
$$

Also, letting $\psi=\psi_{\sigma_{m}}-\psi_{\sigma_{m}}(R)$ and using Eq. 3.3,

$$
\begin{aligned}
\left\|\sigma_{m}-\mathbf{U}_{R} \sigma_{m}\right\|_{H^{1}\left(\Omega_{R}\right)} & =\left\|\nabla^{\perp} \psi-\nabla^{\perp}\left(h_{R} \psi\right)\right\|_{H^{1}\left(\Omega_{R}\right)} \\
& \leq\left\|\left(1-h_{R}\right) \sigma_{m}\right\|_{H^{1}\left(\Omega_{R}\right)}+\left\|\nabla^{\perp} h_{R} \psi\right\|_{H^{1}\left(\Omega_{R}\right)} \\
& \leq\left\|1-h_{R}\right\|_{C^{1}}\left\|\sigma_{m}\right\|_{H^{1}\left(\Omega_{R} \backslash \Omega_{R-1}\right)}+\left\|\nabla h_{R}\right\|_{C^{1}}\|\psi\|_{H^{1}\left(\Omega_{R} \backslash \Omega_{R-1}\right)} \\
& \leq C\left\|\sigma_{m}\right\|_{H^{1}\left(\Omega_{R} \backslash \Omega_{R-1}\right)}+C\|\psi\|_{H^{1}\left(\Omega_{R} \backslash \Omega_{R-1}\right)} \\
& \leq C\left\|\sigma_{m}\right\|_{H^{1}\left(\Omega_{R} \backslash \Omega_{R-1}\right)}+C\|\psi\|_{L^{2}\left(\Omega_{R} \backslash \Omega_{R-1}\right)} .
\end{aligned}
$$

Because $\Omega_{R} \backslash \Omega_{R-1}$ has width 1 and $\psi$ vanishes on its outer boundary, we can apply Poincare's inequality with a constant that is independent of $R$ to give

$$
\|\psi\|_{L^{2}\left(\Omega_{R} \backslash \Omega_{R-1}\right)} \leq C\|\nabla \psi\|_{L^{2}\left(\Omega_{R} \backslash \Omega_{R-1}\right)}=C\left\|\sigma_{m}\right\|_{L^{2}\left(\Omega_{R} \backslash \Omega_{R-1}\right)} .
$$

Thus,

$$
\left\|\sigma_{m}-\mathbf{U}_{R} \sigma_{m}\right\|_{H^{1}\left(\Omega_{R}\right)} \leq C\left\|\sigma_{m}\right\|_{H^{1}\left(\Omega_{R} \backslash \Omega_{R-1}\right)}=C|m|\left\|\sigma_{1}\right\|_{H^{1}\left(\Omega_{R} \backslash \Omega_{R-1}\right)},
$$

which vanishes as $R \rightarrow \infty$ by Eq. 2.5. This gives Eq. 3.4 for $\mathbf{U}_{R}$. Projection into $V_{R}$ gives the closest element in $V_{R}$, so Eq. 3.4 holds for $\mathbf{P}_{V_{R}}$.

Equation 3.5 holds simply because $\mathbf{P}_{V_{R}}$ is an orthogonal projection operator.

To prove Eq. 3.6, let $u=\sigma_{m}+v$ in $E_{m} \cap \dot{H}^{1}\left(\mathbb{R}^{2}\right)$. Then

$$
\begin{aligned}
\left\|\mathbf{P}_{V_{R}} u\right\|_{V_{R}} & \leq\left\|\mathbf{P}_{V_{R}} \sigma_{m}\right\|_{V_{R}}+\left\|\mathbf{P}_{V_{R}} v\right\|_{V_{R}} \leq\left\|\sigma_{m}\right\|_{V_{R}}+\|v\|_{V_{R}} \\
& \leq\left\|\sigma_{m}\right\|_{V}+\|v\|_{V}=|m|\left\|\sigma_{1}\right\|_{V}+\|v\|_{V}=C|m|+\|v\|_{V} \\
& \leq\|u\|_{\mathbb{E}^{1}}+C|m| .
\end{aligned}
$$

Lemma 3.3 $\mathbf{P}_{V_{R}} \sigma_{m}$ is a stationary solution to the Euler equations on $\Omega_{R}$.

Proof Let $\bar{\sigma}_{m}=\mathbf{P}_{V_{R}} \sigma_{m}$. Since $\psi_{\sigma_{m}}$ and $\omega\left(\sigma_{m}\right)$ are radially symmetric, so too must $\psi_{\bar{\sigma}_{m}}$ and $\omega\left(\bar{\sigma}_{m}\right)$ be. But then

$$
\omega\left(\bar{\sigma}_{m} \cdot \nabla \bar{\sigma}_{m}\right)=\bar{\sigma}_{m} \cdot \nabla \omega\left(\bar{\sigma}_{m}\right)=0,
$$

and thus $\bar{\sigma}_{m} \cdot \nabla \bar{\sigma}_{m}=\nabla p$ for some scalar field $p$.

Remark 3.4 Equations 3.4 through 3.6 continue hold if the projection operator $\mathbf{P}_{V_{R}}$ is replaced by the approximate projection operator $\mathbf{U}_{R}$, though a constant factor is introduced on the right-hand sides of Eqs. 3.5 and 3.6. Equations 3.4 through 3.6 also hold with $\mathbf{P}_{V_{R}}$ replaced by $\mathbf{U}_{R}$ and $H^{1}$ replaced by $L^{2}$. This gives control not only on the $H^{1}$-norm but individual control on the $L^{2}$-norm. See Remarks 5.3 and 5.6. 
We will use the operator $\mathbf{P}_{V_{R}}$ in establishing the deterministic expanding domain limit in Sect. 5 and in constructing statistical solutions to $(N S)$ in Sect. 7. For solutions to the Euler equations, we will need the following approximate truncation operator of Definition 3.5 (or we could use projection into $Y\left(\Omega_{R}\right)$ ).

Definition 3.5 Define $\overline{\mathbf{U}}_{R}: \mathbb{Y} \rightarrow \mathbb{Y}\left(\Omega_{R}\right)$ by

$$
\overline{\mathbf{U}}_{R}\left(\sigma_{m}+v\right)=\left.\sigma_{m}\right|_{\Omega_{R}}+T_{R} v,
$$

where $T_{R}: \mathbb{Y}_{0} \rightarrow \mathbb{Y}\left(\Omega_{R}\right)$ is the operator in Lemma 4.2 of [10].

Because $\Omega_{R}$ is a disk, $\left.\sigma_{m}\right|_{\Omega_{R}}$ is in $H_{R}$ and so also in $\mathbb{Y}\left(\Omega_{R}\right)$. This is why $\overline{\mathbf{U}}_{R}: \mathbb{Y} \rightarrow \mathbb{Y}\left(\Omega_{R}\right)$. It is also why if we define $\mathbf{P}_{H_{R}}$ to be the restriction to $\Omega_{R}$ followed by projection into $H_{R}$ that for all $u$ in $\mathbb{E}$,

$$
\mathbf{P}_{H_{R}} u=\sigma(u)+\mathbf{P}_{H_{R}}(u-\sigma(u)) .
$$

It follows from Lemma 4.2 of [10] that for all $u$ in $\mathbb{E}$,

$$
\left\|\mathbf{P}_{H_{R}} u-u\right\|_{L^{2}\left(\Omega_{R}\right)} \rightarrow 0 \text { as } R \rightarrow \infty
$$

Actually, Lemma 4.2 of [10] applies to an approximate projection operator into $H_{R}$, but we are using, as in in $[8,11]$, the fact that projection into $H_{R}$ gives the closest vector field in $H_{R}$ to the vector field being projected.

\section{Weak Deterministic Solutions}

Definition 4.1 (Weak Navier-Stokes Solution) Given viscosity $v>0$, initial velocity $u_{0}$ in $H_{R}$, and forcing $f$ in $L_{l o c}^{2}\left([0, \infty), H_{R}\right), u$ in $L^{2}\left([0, T] ; V_{R}\right)$ with $\partial_{t} u$ in $L^{2}\left([0, T] ; V_{R}^{\prime}\right)$ is a weak solution to the Navier-Stokes equations on $\Omega_{R}$ if $u(0)=u_{0}$ and

$$
\int_{\Omega_{R}} \partial_{t} u \cdot v+\int_{\Omega_{R}}(u \cdot \nabla u) \cdot v+v \int_{\Omega_{R}} \nabla u \cdot \nabla v=(u, f)
$$

for almost all $t$ in $[0, T]$ and for all $v$ in $V_{R}$. A weak solution on $\mathbb{R}^{2}$ is defined for $u_{0}$ in $E_{m}$ with $u$ lying in $L^{2}\left([0, T] ; \dot{H}^{1}\right)$ and $\partial_{t} u$ in $L^{2}\left([0, T] ; V^{\prime}\right)$, and with $(N S)$ holding for all $v$ in $V$.

Definition 4.2 (Weak Euler Solution) Given an initial velocity $u_{0}$ in $\mathbb{Y}\left(\Omega_{R}\right)$ and forcing $f$ in $L_{l o c}^{2}\left([0, \infty), H_{R}\right), u$ in $L^{\infty}\left([0, T] ; H_{R} \cap H^{1}\left(\Omega_{R}\right)\right)$ with $\partial_{t} u$ in $L^{2}\left([0, T] ; V_{R}^{\prime}\right)$ is a weak solution to the Euler equations if $u(0)=u_{0}$ and

$$
\text { (E) } \quad \int_{\Omega_{R}} \partial_{t} u \cdot v+\int_{\Omega_{R}}(u \cdot \nabla u) \cdot v=(f, v)
$$

for almost all $t$ in $[0, T]$ and for all $v$ in $H_{R} \cap H^{1}\left(\Omega_{R}\right)$. A weak solution on $\mathbb{R}^{2}$ is defined for $u_{0}$ in $\mathbb{Y}_{m}$ with $u$ lying in $L^{\infty}\left([0, T] ; \mathbb{Y}_{m}\right)$ and $\partial_{t} u$ in $L^{2}\left([0, T] ; V^{\prime}\right)$, and with $(E)$ holding for all $v$ in $V$.

Note that the test functions always have finite energy, even for solutions in the whole plane. (Test functions in $E_{m}$ would be too large to define the integrals involving the nonlinear terms in $(N S)$ and $(E)$.) 
Given a solution to $(N S)$, there exists a distribution $p$ (tempered, if the solution is in the whole plane) such that

$$
\partial_{t} u+u \cdot \nabla u+\nabla p=v \Delta u+f
$$

equality holding in the sense of distributions. This follows from a result of Poincaré and de Rham that any distribution that is a curl-free vector is the gradient of some scalar distribution.

Given a solution to $(E)$, there exists a pressure $p$ such that

$$
\partial_{t} u+u \cdot \nabla u+\nabla p=f
$$

but we can only interpret $p$ as a distribution when working in the whole plane. Otherwise, we must view $\partial_{t} u+u \cdot \nabla u$ as lying in $H^{-1}\left(\Omega_{R}\right)$ and $p$ as lying in $L^{2}\left(\Omega_{R}\right)$ (Eq. 4.2 follows, for instance, from Remark I.1.9 p. 14 of [13]).

In both Eqs. 4.1 and 4.2 the pressure is unique up to the addition of a function of time. We resolve this ambiguity on $\Omega_{R}$ by requiring that $\int_{\Omega_{R}} p(t)=0$ and on $\mathbb{R}^{2}$ by requiring that $p(t)$ lie in $L^{2}\left(\Omega_{R}\right)$ for almost all $t$ in $[0, T]$.

In referring to solutions on $\Omega_{R}$ we will say solutions for $R$ in $[1, \infty)$ and in referring to solutions on $\mathbb{R}^{2}$ we will say solutions for $R=\infty$.

Theorem 4.3 (1) Assume that $u_{0}$ is in $E_{m} \cap \dot{H}^{1}$. There exists a unique weak solution $(u, p)$ to $(N S)$ in the sense of Definition 4.1 with initial velocity $u_{0}$ for $R=\infty$ and initial velocity $\mathbf{P}_{V_{R}} u_{0}$ for $R$ in $[1, \infty)$, with

$$
u-\sigma_{m} \in L^{\infty}\left([0, T] ; H_{R}\right), \nabla u \in L^{\infty}\left([0, T] ; L^{2}\left(\Omega_{R}\right)\right) .
$$

Moreover, there is a bound on each of these norms that is independent of $R$ in $[1, \infty]$ and that depends continuously on $\left\|u_{0}\right\|_{\mathbb{E}^{1}}$. For $R=\infty$, if $u_{0}$ is in $E_{m} \cap \dot{H}^{1}$ and $\omega(f)$ is in $L^{1}\left([0, T] ; L^{p_{0}} \cap L^{\infty}\right)$ then $\omega(u)$ is in $L^{\infty}\left([0, T] ; L^{p_{0}} \cap L^{\infty}\right)$.

(2) Assume that $u_{0}$ is in $\mathbb{Y}_{m}$ and that $\omega(f)$ is in $L^{1}\left([0, T] ; L^{p_{0}} \cap L^{\infty}\right)$. There exists a unique weak solution $(u, p)$ to $(E)$ in the sense of Definition 4.2 with initial velocity $u_{0}$ for $R=\infty$ and initial velocity $\overline{\mathbf{U}}_{R} u_{0}$ for $R$ in $[1, \infty)$. We have,

$$
\begin{aligned}
u-\sigma_{m} & \in L^{\infty}\left([0, T] ; H_{R}\right), \quad \nabla u \in L^{\infty}\left([0, T] ; L^{2}\left(\Omega_{R}\right)\right), \\
u & \in L^{\infty}\left([0, T] \times \Omega_{R}\right), \quad u \in C\left([0, T] \times \overline{\Omega_{R}}\right), \\
\partial_{t} u & \in L^{\infty}\left([0, T] ; H_{R}\right), \quad \nabla p \in L^{\infty}\left([0, T] ; L^{2}\left(\Omega_{R}\right)\right), \\
\omega(u) & \in L^{\infty}\left([0, T] ; L^{p_{0}} \cap L^{\infty}\left(\Omega_{R}\right)\right),
\end{aligned}
$$

and there is a bound on each of these norms that is independent of $R$ in $[1, \infty]$ and that depends continuously on $\left\|u_{0}\right\|_{\mathbb{Y}}$.

Proof These results are standard for $R<\infty$, except for the independence of the norms on $R$. The independence of the first norm in Eq. 4.3 follows for solutions to $(N S)$ from the energy inequality in Eq. 5.4 along with Eq. 3.4; for the second norm it follows from adapting slightly the proof of this same fact for finite energy in [10]. The stronger bounds for solutions to $(E)$ follow from the vorticity equation for $(E)$. The results for $R=\infty$ are a minor modification of the same results for finite-energy: see, for instance, [2].

Definition 4.4 (Solution operators) Fix $f_{R}$ in $L^{2}\left([0, \infty) ; H_{R}\right)$ and write $f$ for $f_{\infty}$. Let $S_{R}(t)$ be the solution operator for $(N S)$ on $\Omega_{R}$ with $S=S_{\infty}$, and let $\bar{S}_{R}(t)$ be the solution operator for $(E)$ on $\Omega_{R}$ with $\bar{S}=\bar{S}_{\infty}$. 
In application, we will often start with $f$ in $L^{2}([0, \infty) ; H)$ or even time-independent $f$ in $H$ and let $f_{R}=\mathbf{P}_{H_{R}} f$.

$S_{R}(0)$ is the identity operator, as is $\bar{S}_{R}(0)$. For all $t>0, S_{R}$ maps $H_{R} \rightarrow V_{R}$ and $V_{R} \rightarrow V_{R}$ and $S$ maps $E_{m} \rightarrow E_{m} \cap \dot{H}^{1}, E_{m} \cap \dot{H}^{1} \rightarrow E_{m} \cap \dot{H}^{1}, \mathbb{E} \rightarrow \mathbb{E}^{1}$, and $\mathbb{E}^{1} \rightarrow \mathbb{E}^{1}$. For all $t \geq 0, \bar{S}_{R}$ maps $Y\left(\Omega_{R}\right) \rightarrow Y\left(\Omega_{R}\right)$ and $\bar{S}$ maps $\mathbb{Y}_{m} \rightarrow \mathbb{Y}_{m}$ and $\mathbb{Y} \rightarrow \mathbb{Y}$. Each of these maps is continuous.

Observe that $S_{R}(t) \mathbf{P}_{V_{R}} u_{0}=u(t)$ for $R$ in $[1, \infty)$ and $S(t) u_{0}=u(t)$ for $R=\infty$ in part (1) of Theorem 4.3, while $\bar{S}_{R}(t) \overline{\mathbf{U}}_{R} u_{0}=u(t)$ for $R$ in $[1, \infty)$ and $\bar{S}(t) u_{0}=u(t)$ for $R=\infty$ in part (2).

\section{Deterministic Expanding Domain Limit}

First we establish the basic energy equality for deterministic solutions to $(N S)$ in $\Omega_{R}$ and in all of $\mathbb{R}^{2}$. In all of $\mathbb{R}^{2}$, the energy is not finite, so we need to subtract $\sigma_{m}$ from the velocity to produce an "energy" equality. To make these estimates uniform over $R$ in $[1, \infty]$, we need, then, to subtract $\sigma_{m}$ from the velocity for $R<\infty$ as well. Actually, it will be slightly more convenient to subtract

$$
\bar{\sigma}_{m}=\mathbf{P}_{V_{R}} \sigma_{m} \text { for } R \in[1, \infty), \quad \bar{\sigma}_{m}=\sigma_{m} \text { for } R=\infty
$$

instead, but because $\bar{\sigma}_{m} \rightarrow \sigma_{m}$ in the $H^{1}\left(\Omega_{R}\right)$-norm as $R \rightarrow \infty$ by Eq. 3.4 , this amounts to the same thing.

Theorem 5.1 Let $u$ be a solution to $(N S)$ as in Definition 4.1 with initial velocity $u_{0}$ in $H_{R}$ for $R<\infty$ and $u_{0}$ in $E_{m}$ for $R=\infty$. Then

$$
\begin{aligned}
& \left\|\left(u-\bar{\sigma}_{m}\right)(t)\right\|_{L^{2}\left(\Omega_{R}\right)}^{2}+2 v \int_{0}^{t}\left\|\nabla\left(u-\bar{\sigma}_{m}\right)\right\|_{L^{2}\left(\Omega_{R}\right)}^{2} \\
& =\left\|u_{0}-\bar{\sigma}_{m}\right\|_{L^{2}\left(\Omega_{R}\right)}^{2}-2 \int_{0}^{t} \int_{\Omega_{R}}\left(\left(u-\bar{\sigma}_{m}\right) \cdot \nabla \bar{\sigma}_{m}\right) \cdot\left(u-\bar{\sigma}_{m}\right) \\
& -2 v \int_{0}^{t} \int_{\Omega_{R}} \nabla \bar{\sigma}_{m} \cdot \nabla\left(u-\bar{\sigma}_{m}\right)+2 \int_{0}^{t} \int_{\Omega} f_{R} \cdot\left(u-\bar{\sigma}_{m}\right)
\end{aligned}
$$

and

$$
\begin{aligned}
& \left\|\left(u-\bar{\sigma}_{m}\right)(t)\right\|_{L^{2}\left(\Omega_{R}\right)}^{2}+v \int_{0}^{t}\left\|\nabla\left(u-\bar{\sigma}_{m}\right)\right\|_{L^{2}\left(\Omega_{R}\right)}^{2} \\
& \quad \leq\left(\left\|u_{0}-\bar{\sigma}_{m}\right\|_{L^{2}\left(\Omega_{R}\right)}^{2}+C m^{2} v t+\|f\|_{L^{1}\left([0, t] ; L^{2}\right)}^{2}\right)^{1 / 2} e^{(C m+1) t},
\end{aligned}
$$

where $C$ depends only on $\left\|\nabla \sigma_{1}\right\|_{L^{2} \cap L^{\infty}}$.

Proof Assume first that $R<\infty$. Using $u-\bar{\sigma}_{m}$, which is in $V_{R}$ for all $t>0$, as a test function in $(N S)$ gives

$$
\int_{\Omega_{R}} \partial_{t} u \cdot\left(u-\bar{\sigma}_{m}\right)+\int_{\Omega_{R}}(u \cdot \nabla u) \cdot\left(u-\bar{\sigma}_{m}\right)+v \int_{\Omega_{R}} \nabla u \cdot \nabla\left(u-\bar{\sigma}_{m}\right)=\int_{\Omega_{R}} f \cdot\left(u-\bar{\sigma}_{m}\right) .
$$


But,

$$
\int_{\Omega_{R}} \partial_{t} u \cdot\left(u-\bar{\sigma}_{m}\right)=\int_{\Omega_{R}} \partial_{t}\left(u-\bar{\sigma}_{m}\right) \cdot\left(u-\bar{\sigma}_{m}\right)=\frac{1}{2} \frac{d}{d t}\left\|u-\bar{\sigma}_{m}\right\|_{L^{2}\left(\Omega_{R}\right)}^{2}
$$

and

$$
\begin{aligned}
\int_{\Omega_{R}}(u \cdot \nabla u) \cdot\left(u-\bar{\sigma}_{m}\right)= & \int_{\Omega_{R}}\left(u \cdot \nabla\left(u-\bar{\sigma}_{m}\right)\right) \cdot\left(u-\bar{\sigma}_{m}\right) \\
& +\int_{\Omega_{R}}\left(u \cdot \nabla \bar{\sigma}_{m}\right) \cdot\left(u-\bar{\sigma}_{m}\right) \\
= & \frac{1}{2} \int_{\Omega_{R}} u \cdot \nabla\left|u-\bar{\sigma}_{m}\right|^{2}+\int_{\Omega_{R}}\left(\left(u-\bar{\sigma}_{m}\right) \cdot \nabla \bar{\sigma}_{m}\right) \cdot\left(u-\bar{\sigma}_{m}\right) \\
& +\int_{\Omega_{R}}\left(\bar{\sigma}_{m} \cdot \nabla \bar{\sigma}_{m}\right) \cdot\left(u-\bar{\sigma}_{m}\right) .
\end{aligned}
$$

The first integral in the right-hand side above is formally zero because div $u=0$ and $u \cdot \mathbf{n}=0$ on $\partial \Omega_{R}$. More properly, we first observe that the integral is finite. This is because at time $t$ in $[0, T]$ both $u$ and $u-\bar{\sigma}_{m}$ are in $H^{1}\left(\Omega_{R}\right)$ and so in $L^{4}\left(\Omega_{R}\right)$ by Sobolev embedding. But $|\nabla| u-\left.\bar{\sigma}_{m}\right|^{2}|\leq 2| u-\bar{\sigma}_{m}|| \nabla\left(u-\bar{\sigma}_{m}\right) \mid$, and applying Hölder's inequality gives the finiteness of the integral. Approximating by smooth functions and using the dominated convergence theorem shows that the integral is zero. (This is the approach of Lemmas II.1.1 and II.1.3 pp. 108-109 of [13].) Since by Lemma 3.3, $\bar{\sigma}_{m} \cdot \nabla \bar{\sigma}_{m}$ is a gradient, the last integral above vanishes. We conclude that

$$
\int_{\Omega_{R}}(u \cdot \nabla u) \cdot\left(u-\bar{\sigma}_{m}\right)=\int_{\Omega_{R}}\left(\left(u-\bar{\sigma}_{m}\right) \cdot \nabla \bar{\sigma}_{m}\right) \cdot\left(u-\bar{\sigma}_{m}\right) .
$$

For the final term, we observe that

$$
\int_{\Omega_{R}} \nabla u \cdot \nabla\left(u-\bar{\sigma}_{m}\right)=\int_{\Omega_{R}} \nabla\left(u-\bar{\sigma}_{m}\right) \cdot \nabla\left(u-\bar{\sigma}_{m}\right)+\int_{\Omega_{R}} \nabla \bar{\sigma}_{m} \cdot \nabla\left(u-\bar{\sigma}_{m}\right) .
$$

Combining all these equalities gives

$$
\begin{aligned}
\frac{1}{2} \frac{d}{d t}\left\|u-\bar{\sigma}_{m}\right\|_{L^{2}\left(\Omega_{R}\right)}^{2}+v\left\|\nabla\left(u-\bar{\sigma}_{m}\right)\right\|_{L^{2}\left(\Omega_{R}\right)}^{2}= & -\int_{\Omega_{R}}\left(\left(u-\bar{\sigma}_{m}\right) \cdot \nabla \bar{\sigma}_{m}\right) \cdot\left(u-\bar{\sigma}_{m}\right) \\
& -v \int_{\Omega_{R}} \nabla \bar{\sigma}_{m} \cdot \nabla\left(u-\bar{\sigma}_{m}\right) \\
& +\int_{\Omega_{R}} f_{R} \cdot\left(u-\bar{\sigma}_{m}\right) .
\end{aligned}
$$


Integrating in time gives Eq. 5.1. Also, we can bound the right-hand side by

$$
\begin{aligned}
& \left\|\nabla \bar{\sigma}_{m}\right\|_{L^{\infty}\left(\mathbb{R}^{2}\right)}\left\|u-\bar{\sigma}_{m}\right\|_{L^{2}\left(\Omega_{R}\right)}^{2}+v\left\|\nabla \bar{\sigma}_{m}\right\|_{L^{2}\left(\mathbb{R}^{2}\right)}\left\|\nabla\left(u-\bar{\sigma}_{m}\right)\right\|_{L^{2}\left(\Omega_{R}\right)} \\
& \quad+\left\|f_{R}\right\|_{L^{2}\left(\Omega_{R}\right)}\left\|u-\bar{\sigma}_{m}\right\|_{L^{2}\left(\Omega_{R}\right)} \\
& \quad \leq C m\left\|u-\bar{\sigma}_{m}\right\|_{L^{2}\left(\Omega_{R}\right)}^{2}+C m^{2} v+\frac{1}{2}\left\|f_{R}\right\|_{L^{2}\left(\Omega_{R}\right)}^{2}+\frac{v}{2}\left\|\nabla\left(u-\bar{\sigma}_{m}\right)\right\|_{L^{2}\left(\Omega_{R}\right)}^{2},
\end{aligned}
$$

where we used Young's inequality and $\bar{\sigma}_{m}=m \sigma_{1}$. Thus,

$$
\begin{aligned}
& \frac{d}{d t}\left\|u-\bar{\sigma}_{m}\right\|_{L^{2}\left(\Omega_{R}\right)}^{2}+v\left\|\nabla\left(u-\bar{\sigma}_{m}\right)\right\|_{L^{2}\left(\Omega_{R}\right)}^{2} \\
& \quad \leq C m^{2} v+\left\|f_{R}\right\|_{L^{2}\left(\Omega_{R}\right)}^{2}+C\left\|u-\bar{\sigma}_{m}\right\|_{L^{2}\left(\Omega_{R}\right)}^{2} .
\end{aligned}
$$

Integrating in time and applying Gronwall's inequality gives Eq. 5.2.

The energy argument above works equally as well when $R=\infty$ with the exception of the term $(1 / 2) \int_{\mathbb{R}^{2}} u \cdot \nabla\left|u-\bar{\sigma}_{m}\right|^{2}$, which must be handled slightly differently, because at time $t$ in $[0, T]$ we no longer have $u$ in $H^{1}\left(\Omega_{R}\right)$, only in $\dot{H}^{1}\left(\mathbb{R}^{2}\right)$. So we divide the integral in two, writing

$$
\begin{aligned}
\frac{1}{2} \int_{\mathbb{R}^{2}} u \cdot \nabla\left|u-\bar{\sigma}_{m}\right|^{2}= & \frac{1}{2} \int_{\mathbb{R}^{2}}\left(u-\bar{\sigma}_{m}\right) \cdot \nabla\left|u-\bar{\sigma}_{m}\right|^{2} \\
& +\frac{1}{2} \int_{\mathbb{R}^{2}} \bar{\sigma}_{m} \cdot \nabla\left|u-\bar{\sigma}_{m}\right|^{2} .
\end{aligned}
$$

The first integral is finite and, in fact, zero, using the same reasoning as with the similar term for $R<\infty$. The vector field $\bar{\sigma}_{m}$ is in $L^{\infty}\left(\mathbb{R}^{2}\right)$ and $\nabla\left|u-\bar{\sigma}_{m}\right|^{2}$ is in $L^{1}\left(\mathbb{R}^{2}\right)$ since $|\nabla| u-\left.\bar{\sigma}_{m}\right|^{2}|\leq 2| u-\bar{\sigma}_{m}|| \nabla\left(u-\bar{\sigma}_{m}\right) \mid$; hence, the second integral is finite. We then have

$$
\begin{aligned}
\left|\frac{1}{2} \int_{\mathbb{R}^{2}} \bar{\sigma}_{m} \cdot \nabla\right| u-\left.\bar{\sigma}_{m}\right|^{2} \mid & =\lim _{R \rightarrow \infty}\left|\frac{1}{2} \int_{\Omega_{R}} \bar{\sigma}_{m} \cdot \nabla\right| u-\left.\bar{\sigma}_{m}\right|^{2} \mid \\
& =\lim _{R \rightarrow \infty}\left|\frac{1}{2} \int_{\partial \Omega_{R}}\left(\bar{\sigma}_{m} \cdot \mathbf{n}\right)\right| u-\left.\bar{\sigma}_{m}\right|^{2} \mid .
\end{aligned}
$$

This integral vanishes, since $\bar{\sigma}_{m} \cdot \mathbf{n}=0$ on $\partial \Omega_{R}$.

Corollary 5.2 Let $f$ lie in $L^{2}([0, \infty) ; H)$ and let $f_{R}=\mathbf{P}_{H_{R}} f$. Let $u_{0}$ be in $E_{m} \cap \mathbb{E}^{1}$ and let $u$ be a solution to $(N S)$ as in Definition 4.1 with initial velocity $\mathbf{P}_{V_{R}} u_{0}$ when $R$ is in $[1, \infty)$ and initial velocity $u_{0}$ when $R=\infty$. Then for a constant $C$ independent of $R$ and $u_{0}$,

$$
\begin{aligned}
& \left\|\left(u-\bar{\sigma}_{m}\right)(t)\right\|_{L^{2}\left(\Omega_{R}\right)}^{2}+v \int_{0}^{t}\left\|\nabla\left(u-\bar{\sigma}_{m}\right)\right\|_{L^{2}\left(\Omega_{R}\right)}^{2} \\
& \quad \leq\left(\left\|u_{0}-\sigma_{m}\right\|_{V_{R}}^{2}+C m^{2} v t+\|f\|_{L^{1}\left([0, t] ; L^{2}\right)}^{2}\right)^{1 / 2} e^{(C m+1) t} .
\end{aligned}
$$

Proof Apply Theorem 5.1 with initial velocity $\mathbf{P}_{V_{R}} u_{0}=\mathbf{P}_{V_{R}}\left(u_{0}-\sigma_{m}\right)+\mathbf{P}_{V_{R}} \sigma_{m}$ and use Eq. 3.5. 
Remark 5.3 Were we to use an initial velocity of $\mathbf{U}_{R} u_{0}$ instead of $\mathbf{P}_{V_{R}} u_{0}$ in Corollary 5.2 we could replace $\left\|u_{0}-\sigma_{m}\right\|_{V_{R}}$ on the right-hand side of Eq. 5.4 with $C\left\|u_{0}-\sigma_{m}\right\|_{H_{R}}$. See Remark 3.4.

We can control the decay of the tail of solutions to $(N S)$ at time $t$ based, ultimately, on the their decay at time zero:

Lemma 5.4 For all $u_{0}$ in $\mathbb{E}^{1}$,

$$
\left\|S(t) u_{0}-\sigma\left(S(t) u_{0}\right)\right\|_{L^{\infty}\left([0, T] ; L^{2}\left(\Omega_{R}^{C}\right)\right)} \rightarrow 0 \text { as } R \rightarrow \infty
$$

and

$$
\left\|S(t) u_{0}-\sigma\left(S(t) u_{0}\right)\right\|_{L^{2}\left([0, T] ; H^{1}\left(\Omega_{R}^{C}\right)\right)} \rightarrow 0 \text { as } R \rightarrow \infty .
$$

Proof This is a minor adaptation of Lemma 7.1 of [10] to account for infinite-energy, and follows by a standard argument.

Theorem 5.5 Assume that $u_{0}$ lies in $\mathbb{E}^{1}$ and let $u_{R}(t)=S_{R}(t) \mathbf{P}_{V_{R}} u_{0}$ and $u(t)=S(t) u_{0}$. Then

$$
\begin{aligned}
&\left\|u_{R}-u\right\|_{L^{\infty}\left([0, T] ; H^{1}\left(\Omega_{R}\right)\right)} \rightarrow 0 \text { as } R \rightarrow \infty, \\
&\left\|\nabla\left(u_{R}-u\right)\right\|_{L^{2}\left([0, T] ; L^{2}\left(\Omega_{R}\right)\right)} \rightarrow 0 \text { as } R \rightarrow \infty,
\end{aligned}
$$

and

$$
\left\|F(t, u)-F_{R}\left(t, u_{R}\right)\right\|_{L^{2}\left([0, T] ; V_{R}^{\prime}\left(\Omega_{R}\right)\right)} \rightarrow 0 \text { as } R \rightarrow \infty .
$$

In addition, the supremum over all $u_{0}$ in any bounded subset of $\mathbb{E}^{1}$ and over all $R$ in $[1, \infty]$ of each of the quantities,

$$
\begin{aligned}
& \left\|u_{R}-\sigma(u)\right\|_{L^{\infty}\left([0, T] ; L^{2}\left(\Omega_{R}\right)\right)},\left\|\nabla u_{R}\right\|_{L^{2}\left([0, T] ; L^{2}\left(\Omega_{R}\right)\right)}, \\
& \left\|F_{R}\left(t, u_{R}\right)\right\|_{L^{2}\left([0, T] ; V_{R}^{\prime}\left(\Omega_{R}\right)\right)}
\end{aligned}
$$

is finite. (For the definition of $F$ and $F_{R}$ see Eq. 6.2 and the paragraph following it.)

Proof The first two bounds in Eq. 5.8 follow from Eq. 5.4. Equations 5.5 and 5.6 follow from Theorem 8.1 of [10] extended to infinite energy solutions using Eq. 5.4 in place of the standard finite-energy energy bounds.

We now prove Eq. 5.7. We have,

$$
\begin{aligned}
\left\|F(t, u)-F_{R}\left(t, u_{R}\right)\right\|_{V_{R}^{\prime}\left(\Omega_{R}\right) \leq} & \left\|A u(t)-A_{R} u_{R}(t)\right\|_{V_{R}^{\prime}\left(\Omega_{R}\right)} \\
& +\left\|B u(t)-B_{R} u_{R}(t)\right\|_{V_{R}^{\prime}\left(\Omega_{R}\right)}+\left\|f-f_{R}\right\|_{V_{R}^{\prime}\left(\Omega_{R}\right)} .
\end{aligned}
$$

Let $v$ be in $V_{R}$ with $\|v\|_{V_{R}}=1$. Then

$$
\begin{aligned}
& \left|\left(A u(t)-A_{R} u_{R}(t), v\right)\right|=v\left|\left(\Delta u(t)-\Delta u_{R}(t), v\right)\right| \\
& \quad=v\left|\left(\nabla u(t)-\nabla u_{R}(t), \nabla v\right)\right| \leq v\left\|\nabla u(t)-\nabla u_{R}(t)\right\|_{L^{2}\left(\Omega_{R}\right)}\|v\|_{V_{R}} .
\end{aligned}
$$

Thus,

$$
\left\|A u(t)-A_{R}\left(u_{R}\right)(t)\right\|_{L^{2}\left([0, T] ; V_{R}^{\prime}\left(\Omega_{R}\right)\right)} \leq v\left\|\nabla u-\nabla u_{R}\right\|_{L^{2}\left([0, T] ; L^{2}\left(\Omega_{R}\right)\right)},
$$

which vanishes as $R \rightarrow \infty$ by Eq. 5.6. 
For the nonlinear term,

$$
\begin{aligned}
\left|\left(B u(t)-B_{R} u_{R}(t), v\right)\right| & =\left|\left(u(t) \cdot \nabla u(t)-u_{R}(t) \cdot \nabla u_{R}(t), v\right)\right| \\
& =\left|\left(\operatorname{div}\left(u(t) \otimes u(t)-u_{R}(t) \otimes u_{R}(t)\right), v\right)\right| \\
& =\left|\left(u(t) \otimes u(t)-u_{R}(t) \otimes u_{R}(t), \nabla v\right)\right| \\
& \leq\left\|u(t) \otimes u(t)-u_{R}(t) \otimes u_{R}(t)\right\|_{L^{2}\left(\Omega_{R}\right)}\|v\|_{V_{R}} .
\end{aligned}
$$

But,

$$
\begin{aligned}
\left\|u(t)^{i} u(t)^{j}-u_{R}(t)^{i} \otimes u_{R}(t)^{j}\right\|_{L^{2}\left(\Omega_{R}\right)} \leq & \left\|u(t)^{i}\left(u(t)^{j}-u_{R}(t)^{j}\right)\right\|_{L^{2}\left(\Omega_{R}\right)} \\
& +\left\|\left(u(t)^{i}-u_{R}(t)^{i}\right) u_{R}(t)^{j}\right\|_{L^{2}\left(\Omega_{R}\right)} \\
\leq & C\|u(t)\|_{L^{4}\left(\Omega_{R}\right)}\left\|u(t)-u_{R}(t)\right\|_{L^{2}\left(\Omega_{R}\right)} \\
\leq & C\|u(t)\|_{L^{2}\left(\Omega_{R}\right)}^{1 / 2}\|\nabla u(t)\|_{L^{2}\left(\Omega_{R}\right)}^{1 / 2} \\
& \times\left\|u(t)-u_{R}(t)\right\|_{L^{2}\left(\Omega_{R}\right)}^{1 / 2} \times\left\|\nabla\left(u(t)-u_{R}(t)\right)\right\|_{L^{2}\left(\Omega_{R}\right)}^{1 / 2} \\
\leq & C\|\nabla u(t)\|_{L^{2}\left(\Omega_{R}\right)}^{1 / 2}\left\|\nabla\left(u(t)-u_{R}(t)\right)\right\|_{L^{2}\left(\Omega_{R}\right)}^{1 / 2},
\end{aligned}
$$

where we used Ladyzhenskaya's inequality (which gives no dependence on $R$ for the constant $C)$. Thus,

$$
\begin{aligned}
& \left\|B u(t)-B_{R}\left(u_{R}\right)(t)\right\|_{L^{2}\left([0, T] ; V_{R}^{\prime}\left(\Omega_{R}\right)\right)} \\
& \leq C\|\nabla u\|_{L^{1}\left([0, T] ; L^{2}\left(\Omega_{R}\right)\right.}\left\|\nabla\left(u(t)-u_{R}(t)\right)\right\|_{L^{1}\left([0, T] ; L^{2}\left(\Omega_{R}\right)\right.}\left\|\nabla\left(u(t)-u_{R}(t)\right)\right\|_{L^{2}\left([0, T] ; L^{2}\left(\Omega_{R}\right)\right.}, \\
& \times C t\|\nabla u\|_{L^{2}\left([0, T] ; L^{2}\left(\Omega_{R}\right)\right.} \| \nabla(x)
\end{aligned}
$$

which vanishes as $R \rightarrow \infty$ by Eq. 5.6.

For the forcing term,

$$
\left\|f-f_{R}\right\|_{L^{2}\left([0, T] ; V_{R}^{\prime}\left(\Omega_{R}\right)\right)} \leq\left\|f-f_{R}\right\|_{L^{2}\left([0, T] ; H_{R}\left(\Omega_{R}\right)\right)},
$$

which vanishes as $R \rightarrow \infty$ by Eq. 3.8.

From these bounds, Eq. 5.7 follows.

It remains to establish the last bound in Eq. 5.8. But this follows from an argument similar to that we just made to prove Eq. 5.7, using the first two bounds in Eq. 5.8.

Remark 5.6 Together, the limits in Eqs. 5.5 and 5.6 are called the expanding domain limit in [10]. Most of the estimates involved in establishing these limits require only that the initial velocity lie in $H$ (or $\mathbb{E}$ for the infinite-energy extension). The key exception is that the regularity of the pressure is insufficient to complete the argument unless the initial velocity is in $V$ (or $\mathbb{E}^{1}$ for the infinite-energy extension). See, however, Remark 7.2.

Had we used $\mathbf{U}_{R}$ in place of $\mathbf{P}_{V_{R}}$ in defining the initial velocity, the expanding domain limit would still hold (indeed, this is how the limit was established in [10]). An advantage of using $\mathbf{U}_{R}$ is that the resulting bound on the rate of convergence is slightly improved, since $H^{1}$-norms are replaced by $L^{2}$-norms in certain constants that appear in the bound. But this is unimportant in our use of the limit, so we preferred to use $\mathbf{P}_{V_{R}}$, since it has a more natural definition.

Corollary 5.7 For all $u_{0}$ in $\mathbb{E}^{1}$,

$$
\left\|S_{R}(t) \mathbf{P}_{V_{R}} u_{0}-\mathbf{P}_{V_{R}} S(t) u_{0}\right\|_{L^{\infty}\left([0, T] ; H_{R}\right)} \rightarrow 0 \text { as } R \rightarrow \infty
$$


and

$$
\left\|S_{R}(t) \mathbf{P}_{V_{R}} u_{0}-\mathbf{P}_{V_{R}} S(t) u_{0}\right\|_{L^{2}\left([0, T] ; V_{R}\right)} \rightarrow 0 \text { as } R \rightarrow \infty .
$$

Proof By Eqs. 5.5 and 5.6

$$
\lim _{R \rightarrow \infty}\left\|S_{R}(t) \mathbf{P}_{V_{R}} u_{0}-S(t) u_{0}\right\|_{L^{2}\left([0, T] ; V_{R}\right)} \rightarrow 0 \text { as } R \rightarrow \infty .
$$

But by Eq. 3.4,

$$
\begin{aligned}
\left\|\mathbf{P}_{V_{R}} S(t) u_{0}-S(t) u_{0}\right\|_{L^{2}\left([0, T] ; V_{R}\right)} \leq & C\left\|S(t) u_{0}-\sigma\left(S(t) u_{0}\right)\right\|_{L^{2}\left([0, T] ; H^{1}\left(\Omega_{R} \backslash \Omega_{R / 2}\right)\right)} \\
& +T^{1 / 2}|m(u)| \beta(R) \\
\leq & C\left\|S(t) u_{0}-\sigma\left(S(t) u_{0}\right)\right\|_{L^{2}\left([0, T] ; H^{1}\left(\Omega_{R / 2}^{C}\right)\right)} \\
& +T^{1 / 2}|m(u)| \beta(R),
\end{aligned}
$$

which also vanishes as $R \rightarrow \infty$ by Lemma 5.4. Equation 5.10 then follows from the triangle inequality. The proof of Eq. 5.9 is similar.

Remark 5.8 It is only in the proof of Corollary 5.7 where we directly use the uniform decay over time of the tail of the velocity for solutions to $(N S)$. It was, however, already used in the extension of the expanding domain limit from finite to infinite energy energy alluded to in the proof of Theorem 5.5.

Lemma 5.9 For all $u$ in $\mathbb{E}^{1}$.

$$
\left\|F(t, u)-F_{R}\left(t, \mathbf{P}_{V_{R}} u\right)\right\|_{V_{R}^{\prime}\left(\Omega_{R}\right)} \rightarrow 0 \text { as } R \rightarrow \infty,
$$

and the supremum over any bounded subset of $\mathbb{E}^{1}$ and over all $R$ in $[1, \infty]$ of

$$
\left\|F_{R}\left(t, \mathbf{P}_{V_{R}} u\right)\right\|_{V_{R}^{\prime}\left(\Omega_{R}\right)}
$$

is finite.

Proof The proof is the same as that of Eq. 5.7, with no need to introduce the $L^{2}$-norm over $[0, T]$, and using Lemma 3.2 in place of the bounds in Eqs. 5.5 and 5.6.

\section{Definition of Infinite-Energy Statistical Solutions}

Following [7] pp. 264-265, we define a statistical solution to $(N S)$ on $\Omega_{R}$, first defining the space of test functions.

Definition 6.1 The space $\mathcal{T}_{R}$ of test functions, $R<\infty$, is the set of all functions $\Phi: H_{R} \rightarrow \mathbb{R}$ such that $\Phi(u)=\phi\left(\left(u, g_{1}\right), \ldots,\left(u, g_{k}\right)\right)$ for some $\phi$ in $C^{1}\left(\mathbb{R}^{k}\right)$ and some $g_{1}, \ldots, g_{k}$ in $V_{R}$. The Fréchet derivative of such a $\Phi$ is given by

$$
\Phi^{\prime}(u)=\sum_{j=1}^{k} \partial_{j} \phi\left(\left(u, g_{1}\right), \ldots,\left(u, g_{k}\right)\right) g_{j},
$$

which lies in $V_{R}$ since each $g_{j}$ is in $V_{R}$. When $R=\infty$, we require that each of $g_{1}, \ldots, g_{k}$ be compactly supported in $\mathbb{R}^{2}$, so that $\Phi: \mathbb{E} \rightarrow \mathbb{R}$, and we also write $\mathcal{T}$ for $\mathcal{T}_{\infty}$. This is the same class of test functions as for homogeneous solutions in the whole space (Definition 2.3 p. 278 of [7]). 
Observe that because each $\partial_{j} \phi$ is bounded,

$$
\left\|\Phi^{\prime}(u)\right\|_{V_{R}} \leq C(\Phi)
$$

for all $u$ in $V_{R}$.

For $t \geq 0$ and $u$ in $H_{R}$ let

$$
F_{R}(t, u)=f_{R}(t)-v A_{R} u-B_{R}(u),
$$

where $A_{R}$ is the Stokes operator and $B_{R}$ is the classical linear operator associated with the nonlinear term in $(N S)$ on $\Omega_{R}$. (See, for instance, p. 38 of [7].) We also write $F$ for $F_{\infty}, A$ for $A_{\infty}$, and $B$ for $B_{\infty}$.

For $R=\infty$, we will assume for simplicity that $f$ is in $L_{l o c}^{2}([0, \infty) ; H)$; that is, we do not allow infinite forcing.

Definition 6.2 (Statistical solution to $(N S)$ ) Assume that $\mu_{0}$ is a Borel probability measure on $H_{R}$. Then a family,

$$
\mu=\left\{\mu_{t}\right\}_{t \geq 0},
$$

of Borel probability measures on $H_{R}, R<\infty$, is a statistical solution to ( $N S$ ) (SSNS) on $\Omega_{R}$ if each of the following is satisfied:

(1) For all $\Phi$ in $\mathcal{T}_{R}$ and all $t \geq 0$,

$$
\begin{aligned}
\int_{H_{R}} \Phi(u) d \mu_{t}(u)= & \int_{H_{R}} \Phi(u) d \mu_{0}(u) \\
& +\int_{0}^{t} \int_{H_{R}}\left(F_{R}(s, u), \Phi^{\prime}(u)\right) d \mu_{s}(u) d s .
\end{aligned}
$$

(2) For all $t \geq 0$,

$$
\begin{aligned}
& \int_{H_{R}}\|u\|_{L^{2}}^{2} d \mu_{t}(u)+2 v \int_{0}^{t} \int_{H_{R}}\|\nabla u\|_{L^{2}}^{2} d \mu_{s}(u) d s \\
& =\int_{0}^{t} \int_{H_{R}}(f(s), u) d \mu_{s}(u) d s+\int_{H_{R}}\|u\|_{L^{2}}^{2} d \mu_{0}(u) .
\end{aligned}
$$

(3) The map

$$
t \mapsto \int_{H_{R}} \phi(u) d \mu_{t}(u)
$$

is measurable for all $t \geq 0$ and all $\phi$ in $C^{0}\left(H_{R}\right)$.

(4) The map

$$
t \mapsto \int_{H_{R}}\|u\|_{H_{R}}^{2} d \mu_{t}(u)
$$

lies in $L_{l o c}^{\infty}([0, \infty))$. 
(5) The map

$$
t \mapsto \int H_{R}\|\nabla u\|_{L^{2}}^{2} d \mu_{t}(u)
$$

lies in $L_{l o c}^{1}([0, \infty))$.

When $R=\infty$, we make two changes in the definition. First, we replace $H_{R}$ by $\mathbb{E}$ throughout. Second, the energy equality in (2) is replaced by

(2') For all $t \geq 0$,

$$
\begin{aligned}
& \int_{\mathbb{E}}\|u-\sigma(u)\|_{L^{2}}^{2} d \mu_{t}(u)+2 v \int_{0}^{t} \int_{\mathbb{E}}\|\nabla(u-\sigma(u))\|_{L^{2}}^{2} d \mu_{s}(u) d s \\
&= \int_{0}^{t} \int_{\mathbb{E}}(f(s), u-\sigma(u)) d \mu_{s}(u) d s+\int_{\mathbb{E}}\|u-\sigma(u)\|_{L^{2}}^{2} d \mu_{0}(u) \\
&\left.-2 \int_{0}^{t} \int_{\mathbb{E}}((u-\sigma(u)) \cdot \nabla \sigma(u)), u-\sigma(u)\right) d \mu_{s}(u) d s \\
&-2 v \int_{0}^{t} \int_{\mathbb{E}} \nabla \sigma(u) \cdot \nabla(u-\sigma(u)) d \mu_{s}(u) d s,
\end{aligned}
$$

where $\sigma(u)$ is defined in Eq. 2.7.

The following is from Theorems 1.1 and 1.2 Chapter V of [7]:

Theorem 6.3 Let $\mu_{0}$ be as in Definition $6.2, R<\infty$, with kinetic energy

$$
\int_{H_{R}}\|u\|_{H_{R}}^{2} d \mu_{0}(u)<\infty
$$

and assume that $f$ lies in $L_{\text {loc }}^{2}\left([0, \infty) ; H_{R}\right)$. There exists a SSNS, $\mu$, as in Definition 6.2. If the support of $\mu_{0}$ is $\left(H_{R}, V_{R}\right)$-bounded as in Definition 2.1 (meaning that the containment in Eq. 6.4 holds for $t=0)$ and $f$ in $H_{R}$ is time-independent then $\mu_{t}=S_{R}(t) \mu_{0}$ for all $t \geq 0$ is a SSNS. Furthermore, this solution is the unique SSNS satisfying Eqs. 6.3 through 6.5:

$$
\begin{aligned}
& t \mapsto \int_{H_{R}} \varphi(u) d \mu_{t}(u) \text { is continuous on }[0, \infty) \text { for all } \varphi \text { in } C\left(H_{R}^{w}\right), \\
& \operatorname{supp} \mu_{t} \subseteq\left\{u \in V_{R}:\|u\|_{H_{R}} \leq M\right\} \text { for all } t \geq 0 \text { for some } M, \\
& \int_{H_{R}} \Psi(t, u) d \mu_{t}(u)= \int_{H_{R}} \Psi(0, u) d \mu_{0}(u) \\
&+\int_{0}^{t} \int_{H_{R}}\left[\Psi_{s}^{\prime}(s, u)+\left(F(u), \Psi_{u}^{\prime}(s, u)\right)\right] d \mu_{s}(u) d s .
\end{aligned}
$$

$H_{R}^{w}$ is the space $H_{R}$ in the weak topology. In Eq. 6.5, equality holds for all Fréchetdifferentiable continuous real-valued functions on $[0, \infty) \times V_{R}$ (see the discussion following Equation V.1.16 in [7] for more details). 
For statistical solutions to $(E)$, we consider only solutions in the whole plane. For solutions to $(E)$ there is no term involving the Stokes operator, so we define

$$
F(t, u)=f(t)-B(u) .
$$

Definition 6.4 (Statistical solution to $(E)$ in the plane) Assume that $\mu_{0}$ is a Borel probability measure on $\mathbb{E}$. A statistical solution to the Euler equations (SSE) on $\mathbb{E}$ satisfies all the properties of a SSNS in Definition 6.2 for $R=\infty$ except that the terms involving $v$ in property (2') are eliminated.

\section{Construction of Navier-Stokes Solutions}

Let $S(t)$ be the solution operator on $\mathbb{E}$ as in Definition 4.4. Given that we expect the analog of Theorem 6.3 to hold for infinite-energy solutions in $\mathbb{R}^{2}$, we would expect that

$$
\mu_{t}=S(t) \mu_{0}
$$

is the unique SSNS associated to the initial measure $\mu_{0}$ if we assume that the support of the initial Borel probability measure $\mu_{0}$ is $\left(\mathbb{E}, \mathbb{E}^{1}\right.$ )-bounded as in Definition 2.1 . We show that this is, in fact, the case. Our approach will be to use the SSNS on $\Omega_{R}$ and take a limit as $R \rightarrow \infty$ in a careful way to demonstrate that $\mu_{t}$ is a SSNS on all of $\mathbb{R}^{2}$.

We start by defining the initial probability measure $\mu_{0}^{R}$ on $H_{R}$ by

$$
\mu_{0}^{R}(E)=\mu_{0}\left(\mathbf{P}_{V_{R}}^{-1} E\right)
$$

for all Borel measurable subsets $E$ of $H_{R}$. Then $\mu_{0}^{R}$ is a probability measure, for $\mu_{0}^{R}\left(H_{R}\right)=$ $\mu_{0}\left(\mathbf{P}_{V_{R}}^{-1} H_{R}\right)=\mu_{0}(\mathbb{E})=1$. Since we are treating initial probability distributions supported on $\mathbb{E}^{1}$, we use projection into $V_{R}$. When working with SSNSs as weak as those of Definition 6.2, projection into $H_{R}$ would be used instead (though the limiting argument in that case is considerably more involved).

Similarly, we define the forcing term $f_{R}$ in $F_{R}$ by letting

$$
f_{R}=\mathbf{P}_{H_{R}} f,
$$

where $\mathbf{P}_{H_{R}}$ is projection into $H_{R}$. For simplicity, we assume that $f$ is time-independent. Then

$$
\left\|f-f_{R}\right\|_{H_{R}} \rightarrow 0 \text { as } R \rightarrow \infty
$$

from Lemma 4.2 of [10] and the observation that projection into $H_{R}$ gives the closest element in $H_{R}$.

We let $\mu^{R}$ be the associated SSNS on $\Omega_{R}$, so that, by Theorem 6.3,

$$
\mu_{t}^{R}=S_{R}(t) \mu_{0}^{R},
$$

meaning that $S_{R}(t) \mu_{0}^{R}(E)=\mu_{0}^{R}\left(S_{R}^{-1}(t) E\right)$ for any Borel measurable subset $E$ of $H_{R}$.

Let $\Phi$ be in $\mathcal{T}$ as in Definition 6.1. Because each $g_{j}$ is compactly supported, for all sufficiently large $R$, we can define a test function $\Phi_{R}$ in $\mathcal{T}_{R}$ by

$$
\Phi_{R}(v) \stackrel{\text { def }}{=} \phi\left(\left(v,\left.g_{1}\right|_{\Omega_{R}}\right), \ldots,\left(v,\left.g_{k}\right|_{\Omega_{R}}\right)\right)=\Phi\left(\mathcal{E}_{R} v\right)
$$

for all $v$ in $H_{R}$, where $\mathcal{E}_{R} v$ is extension by zero of $v$ in $H_{R}$ to all of $\mathbb{R}^{2}$. It follows that for all $v$ in $H_{R}$,

$$
\mathcal{E}_{R} \Phi_{R}^{\prime}(v)=\Phi^{\prime}\left(\mathcal{E}_{R} v\right)
$$


From now on, we always assume that $R$ is sufficiently large that Eq. 7.4 holds.

For all $u$ in $\mathbb{E}^{1}$,

$$
\begin{aligned}
\left|\Phi_{R}\left(\mathbf{P}_{V_{R}} u\right)-\Phi(u)\right| & =\left|\phi\left(\left(\mathbf{P}_{V_{R}} u,\left.g_{1}\right|_{\Omega_{R}}\right), \ldots,\left(\mathbf{P}_{V_{R}} u,\left.g_{k}\right|_{\Omega_{R}}\right)\right)-\phi\left(\left(u, g_{1}\right), \ldots,\left(u, g_{k}\right)\right)\right| \\
& \leq\|\nabla \phi\|_{L^{\infty}}\left|\left(\mathbf{P}_{V_{R}} u-u, g_{1}\right), \ldots,\left(\mathbf{P}_{V_{R}} u-u, g_{k}\right)\right| \\
& \leq\|\phi\|_{C^{1}}\left\|\mathbf{P}_{V_{R}} u-u\right\|_{L^{2}\left(\Omega_{R}\right)}\left(\left\|g_{1}\right\|_{H}^{2}+\cdots+\left\|g_{k}\right\|_{H}^{2}\right)^{1 / 2} \\
& \leq C\left\|\mathbf{P}_{V_{R}} u-u\right\|_{L^{2}\left(\Omega_{R}\right)} .
\end{aligned}
$$

Thus from Lemma 3.2,

$$
\Phi_{R}\left(\mathbf{P}_{V_{R}} u\right) \rightarrow \Phi(u) \text { as } \mathrm{R} \rightarrow \infty
$$

Similarly, for all $u$ in $\mathbb{E}^{1}$,

$$
\begin{aligned}
\left\|\Phi_{R}^{\prime}\left(\mathbf{P}_{V_{R}} u\right)-\Phi^{\prime}(u)\right\|_{V_{R}} \leq & \sum_{j=1}^{k}|\partial|_{j} \phi\left(\left(\mathbf{P}_{V_{R}} u, g_{1}\right), \ldots,\left(\mathbf{P}_{V_{R}} u, g_{k}\right)\right) \\
& -\partial_{j} \phi\left(\left(u, g_{1}\right), \ldots,\left(u, g_{k}\right)\right)\left\|g_{j}\right\|_{V_{R}} \\
\leq & C \sum_{j=1}^{k} \mu_{j}\left(\left|\left(\mathbf{P}_{V_{R}} u, g_{1}\right), \ldots,\left(\mathbf{P}_{V_{R}} u, g_{k}\right)-\left(u, g_{1}\right), \ldots,\left(u, g_{k}\right)\right|\right) \\
= & C \sum_{j=1}^{k} \mu_{j}\left(\left|\left(\mathbf{P}_{V_{R}} u-u, g_{1}\right), \ldots,\left(\mathbf{P}_{V_{R}} u-u, g_{k}\right)\right|\right) \\
\leq & C \sum_{j=1}^{k} \mu_{j}\left(\left\|\mathbf{P}_{V_{R}} u-u\right\|_{H_{R}}\left(\left\|g_{1}\right\|_{H}^{2}+\cdots+\left\|g_{k}\right\|_{H}^{2}\right)^{1 / 2}\right),
\end{aligned}
$$

where $\mu_{j}$ is the modulus of continuity of $\partial_{j} \phi$. Thus by Lemma 3.2,

$$
\left\|\Phi_{R}^{\prime}\left(\mathbf{P}_{V_{R}} u\right)-\Phi^{\prime}(u)\right\|_{V_{R}} \rightarrow 0 \text { as } R \rightarrow \infty
$$

Before proceeding, we mention one logical simplification that we cannot make. It might seem reasonable to try to show that

$$
\begin{aligned}
S(t) \mu_{0} & =\lim _{R \rightarrow \infty} \mathbf{P}_{V_{R}} \circ S_{R}(t) \circ \mathbf{P}_{V_{R}}^{-1} \mu_{0} \\
& =\lim _{R \rightarrow \infty} \mu_{0} \circ \mathbf{P}_{V_{R}}^{-1} \circ S_{R}(t)^{-1} \circ \mathbf{P}_{V_{R}}
\end{aligned}
$$

by showing that equality holds on any Borel measurable set $E$. We note, however, that if $\mu_{0}$ is supported on a singleton set $E=\left\{u_{0}\right\}$ in $\mathbb{E}, u_{0}$ nonzero, then $\mathbf{P}_{V_{R}}^{-1} \circ S_{R}(t)^{-1} \circ \mathbf{P}_{V_{R}}\left(S_{R}(t) u_{0}\right)$ will in general never equal $u_{0}$. Thus, the right-hand side above applied to $E$ will evaluate to 0 for all $R$, while the left-hand side will evaluate to 1 .

Observe that Eq. 7.8 is equivalent to saying that

$$
\int_{\mathbb{E}} \Phi(u) d \mu_{t}(u)=\lim _{R \rightarrow \infty} \int_{H_{R}} \Phi(u) d v_{t}^{R}(u),
$$


where $v_{t}=\mathbf{P}_{V_{R}} \mu_{t}$ for all test functions $\Phi$ (which are dense in the set of bounded continuous functions). We will prove instead that

$$
\int_{\mathbb{E}} \Phi(u) d \mu_{t}(u)=\lim _{R \rightarrow \infty} \int_{H_{R}} \Phi_{R}(u) d \mu_{t}^{R}(u),
$$

and that similar limits hold for the other integral in Property (1) of Definition 6.2, thus circumventing our difficulty. In this weak sense, the expanding domain limit could be said to hold for statistical solutions.

Theorem 6.3 continues to hold for $R=\infty$ if we impose at the outset the condition that the initial velocity $\mu_{0}$ is supported in $\mathbb{E}^{1}$. This condition is required to allow us to take advantage of the expanding domain limit and related bounds from Sect. 5 (see, however, Remark 7.2). The idea for proving existence is to first assume that the measure has bounded support in $\mathbb{E}^{1}$, apply the results of Sect. 5, which require such support, then use the linearity of properties (1-5) of Definition 6.2 to drop the boundedness assumption. This leads to Theorem 7.1.

Theorem 7.1 Let $\mu_{0}$ be as in Definition 6.2 for $R=\infty$, but supported in $\mathbb{E}^{1}$, and having "energy"

$$
\int_{\mathbb{E}}\|u\|_{\mathbb{E}}^{2} d \mu_{0}(u)<\infty .
$$

Assume that $f$ lies in $L_{l o c}^{2}([0, \infty) ; V)$. There exists a SSNS, $\mu$, as in Definition 6.2. If the support of $\mu_{0}$ is $\left(\mathbb{E}, \mathbb{E}^{1}\right)$-bounded as in Definition 2.1 (meaning that Eq. 7.12 holds for $t=0$ ) and $f$ in $V$ is time-independent then $\mu_{t}=S(t) \mu_{0}$ for all $t \geq 0$ is a SSNS. Furthermore, this solution is the unique SSNS satisfying Eqs. 7.11 through 7.13:

$$
\begin{aligned}
& t \mapsto \int_{\mathbb{E}} \varphi(u) d \mu_{t}(u) \text { is continuous on }[0, \infty) \text { for all } \varphi \text { in } C\left(H^{w}\right), \\
& \operatorname{supp} \mu_{t} \subseteq\left\{u \in \mathbb{E}^{1}:\|u\|_{\mathbb{E}} \leq M(t)\right\} \text { for all } t \geq 0, \\
& \int_{\mathbb{E}} \Psi(t, u) d \mu_{t}(u)= \int_{\mathbb{E}} \Psi(0, u) d \mu_{0}(u) \\
&+\int_{0}^{t} \int_{X}\left[\Psi_{s}^{\prime}(s, u)+\left(F(u), \Psi_{u}^{\prime}(s, u)\right)\right] d \mu_{s}(u) d s .
\end{aligned}
$$

In Eq. 7.12, M is continuous on [0, $\infty)$. In Eq. 7.13, equality holds for all Fréchet-differentiable continuous real-valued functions on $[0, \infty) \times V$.

Proof Existence: Assume first that the initial Borel probability measure $\mu_{0}$ has bounded support in $\mathbb{E}^{1}$, meaning that

$$
\operatorname{supp} \mu_{0} \subseteq\left\{u \in \mathbb{E}^{1}:\|u\|_{\mathbb{E}^{1}} \leq M\right\}, \text { for some } M,
$$

and define $\mu_{t}$ by Eq. 7.1 for $t \geq 0$. By Eq. 4.3 it follows that

$$
\operatorname{supp} \mu_{t} \subseteq\left\{u \in \mathbb{E}^{1}:\|u\|_{\mathbb{E}^{1}} \leq M(t)\right\}
$$

for some continuous function $M$. 
In Theorem 5.5, for initial velocity $u_{0}$ in $\mathbb{E}^{1}$ we defined $u_{R}(t)=S_{R}(t) \mathbf{P}_{V_{R}} u_{0}$ and $u(t)=$ $S(t) u_{0}$. In this proof we will be integrating over all initial velocities in $\mathbb{E}$ and calling the initial velocity $u$, to agree with the notation of [7]. In this notation, Eqs. 5.8 and 5.11 become

$$
\begin{aligned}
& \left\|S_{R}(t) \mathbf{P}_{V_{R}} u-\sigma(u)\right\|_{L^{\infty}\left([0, T] ; L^{2}\left(\Omega_{R}\right)\right)}, \quad\left\|\nabla S_{R}(t) \mathbf{P}_{V_{R}} u\right\|_{L^{2}\left([0, T] ; L^{2}\left(\Omega_{R}\right)\right)}, \\
& \left\|F_{R}\left(t, S_{R}(t) \mathbf{P}_{V_{R}} u\right)\right\|_{L^{2}\left([0, T] ; V_{R}^{\prime}\left(\Omega_{R}\right)\right)}, \quad\left\|F_{R}\left(t, \mathbf{P}_{V_{R}} S(t) u\right)\right\|_{V_{R}^{\prime}\left(\Omega_{R}\right)}
\end{aligned}
$$

are bounded on supp $\mu_{0}$ uniformly over all $R$ in $[1, \infty]$. This will allow us to apply the dominated convergence theorem in several steps in our proof.

Using Eq. 7.4, for all $u$ in $\mathbb{E}^{1}$,

$$
\begin{aligned}
\Phi\left(\lim _{R \rightarrow \infty} S_{R}(t) \mathbf{P}_{V_{R}} u\right) & =\Phi\left(\lim _{R \rightarrow \infty} \mathcal{E}_{R} S_{R}(t) \mathbf{P}_{V_{R}} u\right) \\
& =\lim _{R \rightarrow \infty} \Phi\left(\mathcal{E}_{R} S_{R}(t) \mathbf{P}_{V_{R}} u\right)=\lim _{R \rightarrow \infty} \Phi_{R}\left(S_{R}(t) \mathbf{P}_{V_{R}} u\right)
\end{aligned}
$$

where we used the continuity of $\Phi$. Thus we have,

$$
\begin{aligned}
\int_{\mathbb{E}} \Phi(u) d \mu_{t}(u) & =\int_{\mathbb{E}} \Phi(S(t) u) d \mu_{0}(u) \\
& =\int_{\mathbb{E}} \Phi\left(\lim _{R \rightarrow \infty} S_{R}(t) \mathbf{P}_{V_{R}} u\right) d \mu_{0}(u) \\
& =\int_{\mathbb{E}} \lim _{R \rightarrow \infty} \Phi_{R}\left(S_{R}(t) \mathbf{P}_{V_{R}} u\right) d \mu_{0}(u) \\
& =\lim _{R \rightarrow \infty} \int_{\mathbb{E}} \Phi_{R}\left(S_{R}(t) \mathbf{P}_{V_{R}} u\right) d \mu_{0}(u) \\
& =\lim _{R \rightarrow \infty} \int_{H_{R}} \Phi_{R}\left(S_{R}(t) v\right) d \mu_{0}^{R}(v) \\
& =\lim _{R \rightarrow \infty} \int_{H_{R}} \Phi_{R}(u) d \mu_{t}^{R}(u)
\end{aligned}
$$

giving Eq. 7.10. The first equality follows from Eq. 7.1, since the space of bounded continuous functions is dual to the space of Borel probability measures. The limit in the second equality follows from Theorem 5.5. The third equality follows from Eq. 7.16. The fourth equality follows by the dominated convergence theorem, since $\Phi_{R}$ is uniformly bounded over $R$ in $[1, \infty]$ and $\mu_{0}$ is a finite measure. The fifth equality follows from Lemma 7.3. The sixth and final equality follows in the same way as does the first.

This shows that Eq. 7.10 holds for all $t \geq 0$, so if we can show that

$$
\int_{0}^{t} \int_{\mathbb{E}}\left(F(s, u), \Phi^{\prime}(u)\right) d \mu_{s}(u) d s=\lim _{R \rightarrow \infty} \int_{0}^{t} \int_{H_{R}}\left(F_{R}(s, u), \Phi_{R}^{\prime}(u)\right) d \mu_{s}^{R}(u) d s
$$

then we will have established the first property of Definition 6.2 for $\mu$. 
Toward this end,

$$
\begin{aligned}
& \int_{0}^{t} \int_{\mathbb{E}}\left(F(s, u), \Phi^{\prime}(u)\right) d \mu_{s}(u) d s \\
& =\int_{0}^{t} \int_{\mathbb{E}}\left(F(s, S(s) u), \Phi^{\prime}(S(s) u)\right) d \mu_{0}(u) d s \\
& =\int_{0}^{t} \int_{\mathbb{E}} \lim _{R \rightarrow \infty}\left(F_{R}\left(s, \mathbf{P}_{V_{R}} S(s) u\right), \Phi^{\prime}(S(s) u)\right) d \mu_{0}(u) d s \\
& =\int_{0}^{t} \int_{\mathbb{E}} \lim _{R \rightarrow \infty}\left(F_{R}\left(s, \mathbf{P}_{V_{R}} S(s) u\right), \Phi_{R}^{\prime}\left(\mathbf{P}_{V_{R}} S(s) u\right)\right) d \mu_{0}(u) d s \\
& =\lim _{R \rightarrow \infty} \int_{0}^{t} \int_{\mathbb{E}}\left(F_{R}\left(s, \mathbf{P}_{V_{R}} S(s) u\right), \Phi_{R}^{\prime}\left(\mathbf{P}_{V_{R}} S(s) u\right)\right) d \mu_{0}(u) d s .
\end{aligned}
$$

The first equality follows from Lemma 7.4. The second equality follows from Lemma 5.9 and Eq. 7.15, since $\Phi^{\prime}(S(s) u)$ is bounded and compactly supported-and so also we can view the pairings as being in either the duality between $V$ and $V^{\prime}$ or between $V_{R}$ and $V_{R}^{\prime}$. The third equality follows from Eq. 7.7. The fourth equality follows from the dominated convergence theorem using Eq. 7.15.

We would like to commute the roles of the projection operator and the solution operator in the right-hand side of Eq. 7.18 to allow us to apply Lemma 7.3. To do this, we estimate,

$$
\begin{aligned}
D(s, u)= & \mid\left(F_{R}\left(s, \mathbf{P}_{V_{R}} S(s) u\right), \Phi_{R}^{\prime}\left(\mathbf{P}_{V_{R}} S(s) u\right)\right) \\
& -\left(F_{R}\left(s, S_{R}(s) \mathbf{P}_{V_{R}} u\right), \Phi_{R}^{\prime}\left(S_{R}(s) \mathbf{P}_{V_{R}} u\right)\right) \mid \\
\leq & \left|\left(F_{R}\left(s, \mathbf{P}_{V_{R}} S(s) u\right)-F_{R}\left(s, S_{R}(s) \mathbf{P}_{V_{R}} u\right), \Phi_{R}^{\prime}\left(\mathbf{P}_{V_{R}} S(s) u\right)\right)\right| \\
& +\left|\left(F_{R}\left(s, S_{R}(s) \mathbf{P}_{V_{R}} u\right), \Phi_{R}^{\prime}\left(\mathbf{P}_{V_{R}} S(s) u\right)-\Phi_{R}^{\prime}\left(S_{R}(s) \mathbf{P}_{V_{R}} u\right)\right)\right| \\
\leq & \left\|F_{R}\left(s, \mathbf{P}_{V_{R}} S(s) u\right)-F_{R}\left(s, S_{R}(s) \mathbf{P}_{V_{R}} u\right)\right\|_{V_{R}^{\prime}}\left\|\Phi_{R}^{\prime}\left(\mathbf{P}_{V_{R}} S(s) u\right)\right\|_{V_{R}} \\
& +\left\|F_{R}\left(s, S_{R}(s) \mathbf{P}_{V_{R}} u\right)\right\|_{V_{R}^{\prime}}\left\|\Phi_{R}^{\prime}\left(\mathbf{P}_{V_{R}} S(s) u\right)-\Phi_{R}^{\prime}\left(S_{R}(s) \mathbf{P}_{V_{R}} u\right)\right\|_{V_{R}} .
\end{aligned}
$$

Letting

$$
h(R, u, s):=\left\|\Phi_{R}^{\prime}\left(\mathbf{P}_{V_{R}} S(s) u\right)-\Phi_{R}^{\prime}\left(S_{R}(s) \mathbf{P}_{V_{R}} u\right)\right\|_{V_{R}},
$$

we have

$$
\begin{aligned}
\int_{0}^{t} \int_{\mathbb{E}} D(s, u) d \mu_{0}(u) d s \leq & C \int_{0}^{t} \int_{\mathbb{E}}\left\|F_{R}\left(s, \mathbf{P}_{V_{R}} S(s) u\right)-F_{R}\left(s, S_{R}(s) \mathbf{P}_{V_{R}} u\right)\right\|_{V_{R}^{\prime}} d \mu_{0}(u) d s \\
& +\int_{0}^{t} \int_{\mathbb{E}}\left\|F_{R}\left(s, S_{R}(s) \mathbf{P}_{V_{R}} u\right)\right\|_{V_{R}^{\prime}} h(R, u, s) d \mu_{0}(u) d s .
\end{aligned}
$$


Applying the Cauchy-Schwarz inequality, we can bound the last term by

$$
\begin{aligned}
& \int_{\mathbb{E}}\left\|F_{R}\left(s, S_{R}(s) \mathbf{P}_{V_{R}} u\right)\right\|_{L^{2}\left([0, t] ; V_{R}^{\prime}\right)}\|h(R, u, \cdot)\|_{L^{2}([0, t])} d \mu_{0}(u) \\
& \quad \leq C \int_{\mathbb{E}}\|h(R, u, \cdot)\|_{L^{2}([0, t])} d \mu_{0}(u),
\end{aligned}
$$

using Eq. 7.15.

By Eq. 7.5,

$$
\begin{aligned}
h(R, u, s) & =\left\|\Phi_{R}^{\prime}\left(\mathbf{P}_{V_{R}} S(s) u\right)-\Phi_{R}^{\prime}\left(S_{R}(s) \mathbf{P}_{V_{R}} u\right)\right\|_{V_{R}} \\
& =\left\|\Phi^{\prime}\left(\mathcal{E}_{R} \mathbf{P}_{V_{R}} S(s) u\right)-\Phi^{\prime}\left(\mathcal{E}_{R} S_{R}(s) \mathbf{P}_{V_{R}} u\right)\right\|_{V} .
\end{aligned}
$$

By Eq. 6.1, $\left\|\Phi^{\prime}(v)\right\|_{V} \leq C_{0}$ for all $v$ in $H_{R}$, for some $C_{0}$ independent of $R$. Thus, $h(R, u, \cdot) \leq$ $2 C_{0}$. Also, because $\Phi^{\prime}: H \rightarrow V$ is continuous it follows from Eq. 5.9 that $h(R, u, \cdot) \rightarrow 0$ as $R \rightarrow \infty$ for all $u$ in $\mathbb{E}^{1}$. Hence, for all $u$ in $\mathbb{E}^{1}$,

$$
\|h(R, u, \cdot)\|_{L^{2}([0, t])}^{2}=\int_{0}^{t} h(R, u, s)^{2} d s \rightarrow 0
$$

by the dominated convergence theorem. But then also $\|h(R, u, \cdot)\|_{L^{2}([0, t])} \leq 2 C_{0} t^{1 / 2}$ and applying the dominated convergence theorem again gives

$$
\int_{\mathbb{E}}\|h(R, u, \cdot)\|_{L^{2}([0, t])} \rightarrow 0 \text { as } R \rightarrow \infty .
$$

We conclude that the second term on the right-hand side of Eq. 7.20 vanishes as $R \rightarrow \infty$.

For the first term in the right-hand side of Eq. 7.20,

$$
\begin{aligned}
\left\|F_{R}\left(s, \mathbf{P}_{V_{R}} S(s) u\right)-F_{R}\left(s, S_{R}(s) \mathbf{P}_{V_{R}} u\right)\right\|_{V_{R}^{\prime}} \leq & \left\|F_{R}\left(s, \mathbf{P}_{V_{R}} S(s) u\right)-F(s, S(s) u)\right\|_{V_{R}^{\prime}} \\
& +\left\|F(s, S(s) u)-F_{R}\left(s, S_{R}(s) \mathbf{P}_{V_{R}} u\right)\right\|_{V_{R}^{\prime}} .
\end{aligned}
$$

Since $d \mu_{0}(u) d s$ is a finite measure on $[0, t] \times \mathbb{E}$ and the first term on the right-hand side is both bounded and vanishes as $R \rightarrow \infty$ by Lemma 5.9 and Eq. 7.15, after being integrated over $[0, t] \times \mathbb{E}$ the first term vanishes as $R \rightarrow \infty$. The $L^{2}([0, t])$-norm of the second term on the right-hand side is bounded on the support of $\mu_{0}$ by Eq. 7.15 and vanishes as $R \rightarrow \infty$ by Eq. 5.7; applying the Cauchy-Schwarz inequality followed by the dominated convergence theorem shows that

$$
\begin{aligned}
& \int_{0}^{t} \int_{\mathbb{E}}\left\|F(s, S(s) u)-F_{R}\left(s, S_{R}(s) \mathbf{P}_{V_{R}} u\right)\right\|_{V_{R}^{\prime}} d \mu_{0}(u) d s \\
& \leq t^{1 / 2} \int_{\mathbb{E}}\left\|F(s, S(s) u)-F_{R}\left(s, S_{R}(s) \mathbf{P}_{V_{R}} u\right)\right\|_{L^{2}\left([0, t] ; V_{R}^{\prime}\right)} d \mu_{0}(u)
\end{aligned}
$$

vanishes as $R \rightarrow \infty$. 
We conclude that $D(s, u)$ integrates to zero in the limit as $R \rightarrow \infty$, meaning that

$$
\begin{aligned}
& \int_{0}^{t} \int_{\mathbb{E}}\left(F(s, u), \Phi^{\prime}(u)\right) d \mu_{s}(u) d s \\
& \quad=\lim _{R \rightarrow \infty} \int_{0}^{t} \int_{\mathbb{E}}\left(F_{R}\left(s, S_{R}(s) \mathbf{P}_{V_{R}} u\right), \Phi_{R}^{\prime}\left(S_{R}(s) \mathbf{P}_{V_{R}} u\right)\right) d \mu_{0}(u) d s .
\end{aligned}
$$

Finally, using Lemma 7.3 and Lemma 7.4,

$$
\begin{aligned}
& \int_{0}^{t} \int_{\mathbb{E}}\left(F_{R}\left(s, S_{R}(s) \mathbf{P}_{V_{R}} u\right), \Phi_{R}^{\prime}\left(S_{R}(s) \mathbf{P}_{V_{R}} u\right)\right) d \mu_{0}(u) d s \\
& =\int_{0}^{t} \int_{H_{R}}\left(F_{R}\left(s, S_{R}(s) v\right), \Phi_{R}^{\prime}\left(S_{R}(s) v\right)\right) d \mu_{0}^{R}(v) d s \\
& =\int_{0}^{t} \int_{H_{R}}\left(F_{R}(s, v), \Phi_{R}^{\prime}(v)\right) d \mu_{s}^{R}(v) d s,
\end{aligned}
$$

giving Eq. 7.17, completing the demonstration that property (1) of Definition 6.2 is satisfied for $\mu$.

The other properties in Definition 6.2 follow more easily, using the dominated convergence theorem and the first two bounds in Eq. 5.8. Thus, we have established the existence of a SSNS for $R=\infty$ when the initial probability measure has bounded support in $\mathbb{E}^{1}$. But we can drop this restriction by exploiting the inherent linearity in the definition of a SSNS, as done on p. 318 of [7]. This establishes the existence part of the theorem.

Higher regularity: We now add the assumption that the support of $\mu_{0}$ is $\left(\mathbb{E}, \mathbb{E}^{1}\right)$-bounded. Equations 7.11 and 7.12 follow much as did properties (2) through (5) of Definition 6.2. Adding the assumption that $f$ is time-independent, Eq. 7.13 follows for $R=\infty$ in the same way it does for $R<\infty$.

Uniqueness: The proof of uniqueness for $R<\infty$ on pp. 319-321 of [7] applies with the following two changes: First, in the Galerkin approximation we use a basis for $\mathbb{E}^{1}$ in place of the eigenfunctions of the Stokes operator (the spectrum no longer being discrete). Second, we use the energy bound in Eq. 5.2 for $R=\infty$ in place of the bound involving the eigenvalue, $\lambda_{m}$, of the Stokes operator.

Remark 7.2 It is possible to drop the assumption in Theorem 7.1 that $\mu_{0}$ is supported in $\mathbb{E}^{1}$ and still obtain existence and to weaken the assumption that the support of $\mu_{0}$ is $\left(\mathbb{E}, \mathbb{E}^{1}\right)$ bounded to the support of $\mu_{0}$ being bounded in $\mathbb{E}$ and still obtain uniqueness. The argument relies on using the boundary conditions, $u \cdot \mathbf{n}=\omega(u)=0$ on $\partial \Omega_{R}$, in place of no-slip boundary conditions in solutions to the Navier-Stokes equations. Such boundary conditions allow one to bound the gradient of the pressure in $L^{1}\left([0, T] ; L^{2}\left(\Omega_{R}\right)\right)$ uniformly in $R$ and so obtain the expanding domain limit of Theorem 5.5 assuming only that $u_{0}$ lies in $\mathbb{E}$. Key to bounding the pressure in this way is Lemma 1 of [12].

We used the following two elementary lemmas in the proof of Theorem 7.1. Note that when we say that equality holds between two integrals when the integrands are only Borel measurable, we mean that either both integrals are defined and equal or that both integrals 
are undefined. We state the lemmas this way because in their application we do not always know a priori that the integrands are integrable.

Lemma 7.3 For any Borel measurable function $f$ on $H_{R}$,

$$
\int_{\mathbb{E}} f\left(\mathbf{P}_{V_{R}} u\right) d \mu_{0}(u)=\int_{H_{R}} f(v) d \mu_{0}^{R}(v) .
$$

Proof First observe that $f \circ \mathbf{P}_{V_{R}}$ is Borel measurable on $\mathbb{E}$ because $\mathbf{P}_{V_{R}}$ is Borel measurable (in fact, continuous) and $f$ is Borel measurable, so the left-hand side of Eq. 7.21 is well-defined. When $f=\chi_{E}$, the characteristic function of a Borel measurable subset $E$ of $H_{R}$,

$$
\int_{\mathbb{E}} f\left(\mathbf{P}_{V_{R}} u\right) d \mu_{0}(u)=\mu_{0}\left(\mathbf{P}_{V_{R}}^{-1} E\right)=\mu_{0}^{R}(E)=\int_{H_{R}} f(v) d \mu_{0}^{R}(v) .
$$

Equation 7.21 then holds for simple functions by linearity, for nonnegative functions by the monotone convergence theorem, and hence for all Borel measurable functions.

Lemma 7.4 For any function $f$ that is Borel measurable on $H_{R}$,

$$
\int_{H_{R}} f(u) d \mu_{t}^{R}(u)=\int_{H_{R}} f\left(S_{R}(t) u\right) d \mu_{0}^{R}(u) .
$$

When $f$ is Borel measurable on $X$,

$$
\int_{X} f(u) d \mu_{t}(u)=\int_{X} f(S(t) u) d \mu_{0}(u) .
$$

Proof As in the proof of Lemma 7.3, equality holds for simple functions, then nonnegative functions, then all Borel measurable functions.

\section{Construction of Euler Solutions}

We construct infinite-energy statistical solutions to the Euler equations by making a vanishing viscosity argument, using the infinite-energy statistical solutions to the Navier-Stokes equations that we constructed in Sect. 7.

For initial velocities as in Theorem 7.1, we have the following for SSNSs:

Theorem 8.1 Assume that the support of the initial velocity $\mu_{0}$ for a SSNS with $R=\infty$ is bounded in $\mathbb{Y}$ as in Definition 2.1 and that $f$ is time-independent and lies in $\mathbb{Y}_{0}$. Then the SSNS also satisfies

$$
\operatorname{supp} \mu_{t} \subseteq\left\{u \in \mathbb{Y}:\|u\|_{\mathbb{Y}} \leq M(t)\right\},
$$

for a continuous function $M$ independent of $v$, and for all $p$ in $\left[p_{0}, \infty\right]$,

$$
\int_{\mathbb{E}}\|\omega(u)\|_{L^{p}} d \mu_{t}(u) \leq \int_{\mathbb{E}}\|\omega(u)\|_{L^{p}} d \mu_{0}(u)+\int_{0}^{t}\|\omega(f(s))\|_{L^{p}} d s .
$$


Proof It is a standard result that

$$
\|\omega(S(t) u)\|_{L^{p}} \leq\|\omega(u)\|_{L^{p}}+\int_{0}^{t}\|\omega(f(t))\|_{L^{p}}
$$

for all $u$ in $\mathbb{Y}_{0}$. To prove it for $p=r / q$ in lowest terms, with $r$ even, one takes the vorticity of Eq. 4.1, $\partial_{t} \omega+u \cdot \nabla \omega=v \Delta \omega+\omega(f)$, multiplies both sides by $\omega^{p-1}$, and integrates over space and time formally to give

$$
\|\omega(t)\|_{L^{p}}^{p}+p(p-1) \int_{0}^{t}\left\|\omega^{p / 2-1} \nabla \omega\right\|_{L^{2}\left(\mathbb{R}^{2}\right)}^{2}=\left\|\omega_{0}\right\|_{L^{p}}^{p}+p \int_{0}^{t}\left(\omega(f), \omega^{p-1}\right) .
$$

An approximation and smoothing argument is required to establish the equality rigorously, and it then follows for all $p$ in $\left[p_{0}, \infty\right]$ by the continuity of the $L^{p}$ norm as a function of $p$. Applying Hölder's inequality gives Eq. 8.3.

Now assume that $u=\sigma_{m}+v$ is in $\mathbb{Y}_{m}$. Then $\partial_{t} u=\partial_{t} v$ and $\Delta u=\Delta v$ on $\Omega_{1}^{C}$, where $\Delta \sigma_{m}$ vanishes. Thus, the only additional complication in the argument above is the presence of the additional term $\left(\sigma_{m} \cdot \nabla \omega, \omega^{p-1}\right)=(1 / p)\left(\sigma_{m}, \nabla \omega^{p}\right)$. But this vanishes formally by the divergence theorem, since $\sigma_{m} \cdot \mathbf{n}=0$ on $\partial \Omega_{R}$, hence this term need not be accounted for in the approximation and smoothness argument.

Integrating Eq. 8.3 over $\mathbb{E}$ gives

$$
\int_{\mathbb{E}}\|\omega(S(t) u)\|_{L^{p}} d \mu_{0}(u) \leq \int_{\mathbb{E}}\|\omega(u)\|_{L^{p}} d \mu_{0}(u)+\int_{0}^{t}\|\omega(f(t))\|_{L^{p}} .
$$

(The last term has no dependence on $u$ so the integral over $\mathbb{E}$ disappears, $\mu_{0}$ being a probability measure.) But $\|\omega(\cdot)\|_{L^{p}}: \mathbb{Y} \rightarrow[0, \infty)$ is a bounded continuous function on supp $\mu_{0}$ so Eq. 8.2 follows from $\mu_{t}=S(t) \mu_{0}$, and Eq. 8.1 follows from Eq. 8.2.

Theorem 8.2 Assume that $\mu_{0}$ is supported in $\mathbb{Y}$ with

$$
\int_{\mathbb{E}}\|u\|_{\mathbb{E}}^{2} d \mu_{0}(u)<\infty,
$$

and assume that $f$ is time-independent and lies in $\mathbb{Y}_{0}$. There exists a SSE, $\mu$, as in Definition 6.4. One such solution is $\mu_{t}=\bar{S}(t) \mu_{0}$ for all $t \geq 0$, where $\bar{S}(t)$ is the solution operator for the two-dimensional Euler equations in $\mathbb{R}^{2}$ as in Definition 4.4. Furthermore, if the support of $\mu_{0}$ is bounded in $\mathbb{Y}$ as in Definition 2.1 and $f$ is time-independent then this solution satisfies Eq. 8.1 for some function M continuous on $[0, \infty)$ and Eq. 8.2.

Proof Assume first that the support of $\mu_{0}$ is bounded in $\mathbb{Y}$. Define $\bar{\mu}_{t}=\bar{S}(t) \mu_{0}$, and let $\mu$ be the unique SSNS for $R=\infty$ given by Theorem 7.1 with the same forcing and initial data as for the Euler equations. Let $\Phi=\phi\left(\left(u, g_{1}\right), \ldots,\left(u, g_{k}\right)\right)$ lie in $\mathcal{T}$. Then $g_{1}, \ldots, g_{k}$ are in $V$ and

$$
\begin{gathered}
\Phi^{\prime}(u)=\sum_{j=1}^{k} \partial_{j} \phi\left(\left(u, g_{1}\right), \ldots,\left(u, g_{k}\right)\right) g_{j} \in V, \\
\nabla \Phi^{\prime}(u)=\sum_{j=1}^{k} \partial_{j} \phi\left(\left(u, g_{1}\right), \ldots,\left(u, g_{k}\right)\right) \nabla g_{j} \in L^{2},
\end{gathered}
$$


with

$$
\left\|\Phi^{\prime}(u)\right\|_{V} \leq C, \quad\left\|\nabla \Phi^{\prime}(u)\right\|_{L^{2}} \leq C
$$

for some constant $C$ independent of $u$ in $\mathbb{E}$.

Now,

$$
\int_{\mathbb{Y}} \Phi(u) d \mu_{t}(u)=\int_{\mathbb{Y}} \Phi(u) d \mu_{0}(u)+\int_{0}^{t} \int_{\mathbb{Y}}\left(F(s, u), \Phi^{\prime}(u)\right) d \mu_{s}(u) d s
$$

so, using $F=f-v A u-B u$ and $\bar{F}=f-B u$,

$$
\begin{aligned}
\int_{\mathbb{Y}} & \Phi(u) d \mu_{t}(u)-\int_{\mathbb{Y}} \Phi(u) d \bar{\mu}_{0}(u)-\int_{0} \int_{\mathbb{Y}}\left(\bar{F}(s, u), \Phi^{\prime}(u)\right) d \bar{\mu}_{s}(u) d s \\
= & \int_{\mathbb{Y}} \Phi(u) d\left(\mu_{0}-\bar{\mu}_{0}\right)(u)+\int_{0}^{t} \int_{\mathbb{Y}}\left(F(s, u)-\bar{F}(s, u), \Phi^{\prime}(u)\right) d \mu_{s}(u) d s \\
& -\int_{0}^{t} \int_{\mathbb{Y}}\left(B u, \Phi^{\prime}(u)\right) d\left(\mu_{s}-\bar{\mu}_{s}\right)(u) d s \\
= & \int_{\mathbb{Y}} \Phi_{(u)} d\left(\mu_{0}-\bar{\mu}_{0}\right)(u)-v \int_{0}^{t} \int_{\mathbb{Y}}\left(A u, \Phi^{\prime}(u)\right) d \mu_{s}(u) d s \\
- & \int_{0}^{t} \int_{\mathbb{Y}}\left(B u, \Phi^{\prime}(u)\right) d\left(\mu_{s}-\bar{\mu}_{s}\right)(u) d s .
\end{aligned}
$$

But $\mu_{0}=\bar{\mu}_{0}$, so

$$
\begin{aligned}
\int_{\mathbb{Y}} \Phi(u) d\left(\mu_{t}-\bar{\mu}_{t}\right)(u)=-v \int_{0}^{t} \int_{\mathbb{Y}}\left(A u, \Phi^{\prime}(u)\right) d \mu_{s}(u) d s \\
-\int_{0}^{t} \int_{\mathbb{Y}}\left(B u, \Phi^{\prime}(u)\right) d\left(\mu_{s}-\bar{\mu}_{s}\right)(u) d s .
\end{aligned}
$$

We have,

$$
\left(B u, \Phi^{\prime}(u)\right)=\left(u \cdot \nabla u, \Phi^{\prime}(u)\right)
$$

and

$$
\left(A u, \Phi^{\prime}(u)\right)=-\left(\Delta u, \Phi^{\prime}(u)\right)=\left(\nabla u, \nabla \Phi^{\prime}(u)\right),
$$


since $\Phi^{\prime}(u)$ is in $V$. Thus,

$$
\begin{aligned}
\int_{\mathbb{Y}} \Phi(u) d\left(\mu_{t}-\bar{\mu}_{t}\right)(u)= & -v \int_{0}^{t} \int_{\mathbb{Y}}\left(\nabla u, \nabla \Phi^{\prime}(u)\right) d \mu_{s}(u) d s \\
& -\int_{0}^{t} \int_{\mathbb{Y}}\left(u \cdot \nabla u, \Phi^{\prime}(u)\right) d\left(\mu_{s}-\bar{\mu}_{s}\right)(u) d s .
\end{aligned}
$$

We have,

$$
\int_{\mathbb{Y}}\left(\nabla u, \nabla \Phi^{\prime}(u)\right) d \mu_{s}(u) \leq C \int_{\mathbb{Y}}\|\nabla u\|_{L^{2}} d \mu_{s}(u) \leq C,
$$

where we used Eq. 8.4 followed by Eq. 8.2 and the boundedness of the support of $\mu_{0}$ in $\mathbb{Y}$. The same bound holds when integrating against $\bar{\mu}_{s}$. Thus,

$$
\left|\int_{\mathbb{Y}} \Phi(u) d\left(\mu_{t}-\bar{\mu}_{t}\right)(u)\right| \leq R v t+\left|\int_{0}^{t} \int_{\mathbb{Y}}\left(u \cdot \nabla u, \Phi^{\prime}(u)\right) d\left(\mu_{s}-\bar{\mu}_{s}\right)(u) d s\right|,
$$

where $R$ is proportional to the right-hand side of Eq. 8.2, which we note increases with time unless there is zero forcing.

For any Borel measurable function $G$ on $H$,

$$
\begin{aligned}
\int_{\mathbb{Y}} G(u) d\left(\mu_{s}-\bar{\mu}_{s}\right)(u) & =\int_{\mathbb{Y}} G(u) d \mu_{s}(u)-\int_{\mathbb{Y}} G(u) d \bar{\mu}_{s}(u) \\
& =\int_{\mathbb{Y}} G(S(s) u) d \mu_{0}(u)-\int_{\mathbb{Y}} G(\bar{S}(s) u) d \bar{\mu}_{0}(u) \\
& =\int_{\mathbb{Y}}\left(G\left(S(s) u_{0}\right)-G\left(\bar{S}(s) u_{0}\right)\right) d \mu_{0}\left(u_{0}\right) \\
& =\int_{\mathbb{Y}}(G(u(s))-G(\bar{u}(s))) d \mu_{0}\left(u_{0}\right) .
\end{aligned}
$$

In the last integral, we are defining $u(t)$ and $\bar{u}(t)$ to be $S(t) u_{0}$ and $\bar{S}(t) u_{0}$, respectively. These are the solutions to $(N S)$ and $(E)$ given the initial velocity $u_{0}$. (The support of $\mu_{0}$ lying in $\mathbb{Y}$ insures that $\bar{S}(t) u_{0}$ is well-defined and continuous for $\mu_{0}$-almost all $u_{0}$.)

Thus,

$$
\begin{aligned}
& \int_{\mathbb{Y}}\left(u \cdot \nabla u, \Phi^{\prime}(u)\right) d\left(\mu_{s}-\bar{\mu}_{s}\right)(u) \\
& \quad=\int_{\mathbb{Y}}\left[\left(u(s) \cdot \nabla u(s), \Phi^{\prime}(u(s))-\left(\bar{u}(s) \cdot \nabla \bar{u}(s), \Phi^{\prime}(\bar{u}(s))\right)\right] d \mu_{0} .\right.
\end{aligned}
$$


Letting $w=u-\bar{u}$, we have

$$
\begin{aligned}
\left(u \cdot \nabla u, \Phi^{\prime}(u)\right)-\left(\bar{u} \cdot \nabla \bar{u}, \Phi^{\prime}(\bar{u})\right)= & \left(u \cdot \nabla w, \Phi^{\prime}(u)\right) \\
& +\left(u \cdot \nabla \bar{u}, \Phi^{\prime}(u)-\Phi^{\prime}(\bar{u})\right)+\left(w \cdot \nabla \bar{u}, \Phi^{\prime}(\bar{u})\right) \\
= & -\left(u \cdot \nabla \Phi^{\prime}(u), w\right)+\left(u \cdot \nabla \bar{u}, \Phi^{\prime}(u)-\Phi^{\prime}(\bar{u})\right) \\
& +\left(w \cdot \nabla \bar{u}, \Phi^{\prime}(\bar{u})\right),
\end{aligned}
$$

so

$$
\begin{aligned}
& \mid\left(u(s) \cdot \nabla u(s), \Phi^{\prime}(u(s))-\left(\bar{u}(s) \cdot \nabla \bar{u}(s), \Phi^{\prime}(\bar{u}(s))\right) \mid\right. \\
& \leq\|u(s)\|_{L^{\infty}}\left\|\nabla \Phi^{\prime}(u(s))\right\|_{L^{2}}\|w(s)\|_{H} \\
& \quad+\|u(s)\|_{L^{\infty}}\|\nabla \bar{u}(s)\|_{L^{2}}\left\|\Phi^{\prime}(u(s))-\Phi^{\prime}(\bar{u}(s))\right\|_{H} \\
& \quad+\|w(s)\|_{H}\|\nabla \bar{u}(s)\|_{L^{2}}\left\|\Phi^{\prime}(\bar{u}(s))\right\|_{L^{\infty}} .
\end{aligned}
$$

Now, Eq. 8.3 holds for solutions to $(E)$ : it can be derived as for $(N S)$ or by viewing $(E)$ as a non-homogeneous transport equation for the vorticity. Since supp $\mu_{0}$ is bounded in $\mathbb{Y}$, it follows from Eq. 8.3 that $u$ and $\bar{u}$ are bounded in the $L^{\infty}\left([0, T] \times \mathbb{R}^{2}\right)$-norm uniformly over supp $\mu_{0}$, as is $\nabla \bar{u}$ in the $L^{\infty}\left([0, T] ; L^{2}\right)$-norm. This is discussed more fully in [2] or [9], where it is shown, moreover, that there exists a continuous function $\rho:[0, \infty) \times[0, \infty) \rightarrow[0, \infty)$, nondecreasing in $t$, with $\rho(0, t)=0$ for all $t \geq 0$, such that for all $t>0$,

$$
\|w(t)\|_{H} \leq \rho(v, t)
$$

(For sufficiently small $v t, \rho(v, t)=(C v t)^{(1 / 2) e^{-C t}}$. )

Also,

$$
\begin{aligned}
& \left\|\Phi^{\prime}(u(s))-\Phi^{\prime}(\bar{u}(s))\right\|_{H} \\
& \quad \leq \sum_{j=1}^{k}\left|\partial_{j} \phi\left(\left(u(s), g_{1}\right), \ldots,\left(u(s), g_{k}\right)\right)-\partial_{j} \phi\left(\left(u(s), g_{1}\right), \ldots,\left(u(s), g_{k}\right)\right)\right|\left\|g_{j}\right\|_{H} .
\end{aligned}
$$

Now,

$$
\left|\left(u(s), g_{j}\right)-\left(\bar{u}(s), g_{j}\right)\right| \leq\|w(s)\|_{H}\left\|g_{j}\right\|_{H} \leq \rho(v, s)\left\|g_{j}\right\|_{H},
$$

so since each $\partial_{j} \phi$ is continuous, it follows that

$$
\left\|\Phi^{\prime}(u(s))-\Phi^{\prime}(\bar{u}(s))\right\|_{H} \rightarrow 0 \text { as } v \rightarrow 0 \text { uniformly over }[0, T] .
$$

Combining all these facts shows that

$$
\int_{0}^{t} \int_{\mathbb{Y}}\left(u \cdot \nabla u, \Phi^{\prime}(u)\right) d\left(\mu_{s}-\bar{\mu}_{s}\right)(u) \rightarrow 0 \text { as } v \rightarrow 0
$$

and hence that

$$
\lim _{\nu \rightarrow 0} \int_{\mathbb{Y}} \Phi(u) d \mu_{t}(u)=\int_{\mathbb{Y}} \Phi(u) d \bar{\mu}_{0}(u)+\int_{0}^{t} \int_{\mathbb{Y}}\left(\bar{F}(s, u), \Phi^{\prime}(u)\right) d \bar{\mu}_{s}(u) d s .
$$


On the other hand,

$$
\begin{aligned}
\lim _{v \rightarrow 0} \int_{\mathbb{Y}} \Phi(u) d \mu_{t}(u) & =\lim _{\nu \rightarrow 0} \int_{\mathbb{Y}} \Phi(S(t) u) d \mu_{0}(u) \\
& =\int_{\mathbb{Y}} \lim _{\nu \rightarrow 0} \Phi(S(t) u) d \mu_{0}(u)=\int_{\mathbb{Y}} \Phi(\bar{S}(t) u) d \mu_{0}(u) \\
& =\int_{\mathbb{Y}} \Phi(u) d \bar{\mu}_{t}(u) .
\end{aligned}
$$

In the second equality we used the dominated convergence theorem. For the third equality, we used

$$
\begin{aligned}
& |\Phi(S(t) u)-\Phi(\bar{S}(t) u)| \\
& \quad=\left|\phi\left(\left(S(t) u, g_{1}\right), \ldots,\left(S(t) u, g_{k}\right)\right)-\phi\left(\left(\bar{S}(t) u, g_{1}\right), \ldots,\left(\bar{S}(t) u, g_{k}\right)\right)\right| \\
& \quad \leq\|\nabla \phi\|_{L^{\infty}}\left|\left(\left(S(t) u, g_{1}\right), \ldots,\left(S(t) u, g_{k}\right)\right)-\left(\left(\bar{S}(t) u, g_{1}\right), \ldots,\left(\bar{S}(t) u, g_{k}\right)\right)\right| \\
& \quad \leq C\left|\left(\left(S(t) u-\bar{S}(t) u, g_{1}\right), \ldots,\left(S(t) u-\bar{S}(t) u, g_{k}\right)\right)\right| \\
& \quad \leq C\|S(t) u-\bar{S}(t) u\|_{H} \leq C \rho(v, t) \rightarrow 0 \text { as } v \rightarrow 0,
\end{aligned}
$$

the last inequality just being another way of writing Eq. 8.6. Hence, the right-hand sides of Eqs. 8.7 and 8.8 are equal, establishing the first property in Definition 6.4.

Equations 8.1 and 8.2 follow as in the proof of Theorem 8.1.

As in the proof of Theorem 7.1, we can drop the restriction that the support of $\mu_{0}$ is bounded in $\mathbb{Y}$ by exploiting the inherent linearity in the definition of a SSE, as done on p. 318 of [7]. The remaining properties in Definition 6.4 follow using the dominated convergence theorem in a manner similar to what we did above.

The proof of Theorem 8.2 shows that

$$
\int_{\mathbb{Y}} \Phi(u) d \mu_{t}(u) \rightarrow \int_{\mathbb{Y}} \Phi(u) d \bar{\mu}_{t}(u) \text { as } v \rightarrow 0 .
$$

Since the space $\mathcal{T}$ of test functions is dense in the space of all bounded continuous functions on $\mathbb{E}$, it follows that $\mu \rightarrow \bar{\mu}$ as measures as $v \rightarrow 0$; that is, the vanishing viscosity limit holds for statistical solutions to the Navier-Stokes and Euler equations.

Acknowledgements The author was supported in part by NSF grant DMS-0705586 during the period of this work.

Open Access This article is distributed under the terms of the Creative Commons Attribution Noncommercial License which permits any noncommercial use, distribution, and reproduction in any medium, provided the original author(s) and source are credited.

\section{References}

1. Ben-Artzi, M.: Global solutions of two-dimensional Navier-Stokes and Euler equations. Arch. Ration. Mech. Anal. 128(4), 329-358 (1994)

2. Chemin, J.-Y.: A remark on the inviscid limit for two-dimensional incompressible fluids. Comm. Partial Differ. Equat. 21(11-12), 1771-1779 (1996) 
3. Chemin, J.-Y.: Perfect Incompressible Fluids, vol. 14 of Oxford Lecture Series in Mathematics and its Applications. The Clarendon Press Oxford University Press, New York (1998) (Translated from the 1995 French original by Isabelle Gallagher and Dragos Iftimie)

4. Constantin, P., Ramos, F.: Inviscid limit for damped and driven incompressible Navier-Stokes equations in $\mathbb{R}^{2}$. Comm. Math. Phys. 275(2), 529-551 (2007)

5. Foiaş, C.: Statistical study of Navier-Stokes equations. I, II. Rend. Sem. Mat. Univ. Padova 48, 219-348 (1972)

6. Foiaş, C.: Statistical study of Navier-Stokes equations. I, II. Rend. Sem. Mat. Univ. Padova 49, 9-123 (1973)

7. Foias, C., Manley, O., Rosa, R., Temam, R.: Navier-Stokes Equations and Turbulence, vol. 83 of Encyclopedia of Mathematics and its Applications. Cambridge University Press, Cambridge (2001)

8. Iftimie, D., Kelliher, J.P.: Remarks on the vanishing obstacle limit for a $3 \mathrm{~d}$ viscous incompressible fluid. Proc. Am. Math. Soc. 137(2), 685-694 (2009)

9. Kelliher, J.P.: The inviscid limit for two-dimensional incompressible fluids with unbounded vorticity. Math. Res. Lett. 11(4), 519-528 (2004)

10. Kelliher, J.P.: Expanding domain limit for incompressible fluids in the plane. Comm. Math. Phys. 278(3), 753-773 (2008)

11. Kelliher, J.P., Lopes Filho, M.C., Nussenzveig Lopes, H.J.: Vanishing viscosity limit for an expanding domain in space. Ann. Inst. Henri Poincaré (C). doi:10.1016/j.anihpc.2009.07.007

12. Kim, N.: Large friction limit and the inviscid limit of 2-D Navier-Stokes equations under Navier friction condition. SIAM Math. Anal., to appear

13. Temam, R.: Navier-Stokes Equations, Theory and Numerical Analysis. AMS Chelsea Publishing, Providence, RI (2001) (Reprint of the 1984 edn)

14. Yudovich, V.I.: Non-stationary flows of an ideal incompressible fluid. Z̆. Vyčisl. Mat. i Mat. Fiz. 3:10321066 (1963) (Russian)

15. Yudovich, V.I.: Uniqueness theorem for the basic nonstationary problem in the dynamics of an ideal incompressible fluid. Math. Res. Lett. 2(1), 27-38 (1995) 